OPEN ACCESS

Edited by:

Yuhei Nishimura,

Mie University Graduate School

of Medicine, Japan

Reviewed by:

Sang-Bing Ong,

National University of Singapore,

Singapore

Jason N. Peart,

Griffith University, Australia

Lewis J. Watson,

University of Pikeville, United States

*Correspondence:

Ping Liu

330632664@qq.com

Specialty section:

This article was submitted to

Experimental Pharmacology and Drug

Discovery,

a section of the journal

Frontiers in Pharmacology

Received: 07 November 2017

Accepted: 31 January 2018

Published: 21 February 2018

Citation:

Zhang $R$, Han D, Li Z, Shen C, Zhang Y, Li J, Yan G, Li S, Hu B, Li J and Liu P (2018) Ginkgolide C

Alleviates Myocardial

Ischemia/Reperfusion-Induced Inflammatory Injury via Inhibition

of CD40-NF- $\kappa B$ Pathway.

Front. Pharmacol. 9:109.

doi: 10.3389/fphar.2018.00109

\section{Ginkgolide C Alleviates Myocardial Ischemia/Reperfusion-Induced Inflammatory Injury via Inhibition of CD40-NF-кB Pathway}

\author{
Rui Zhang', Dan Han'2, Zhenyu Li', Chengwu Shen', Yahui Zhang', Jun Li', \\ Genquan Yan', Shasha $\mathrm{Li}^{1}$, Bo Hu${ }^{3}$, Jiangbing $\mathrm{Li}^{4}$ and Ping Liu${ }^{1 *}$
}

\footnotetext{
'Department of Pharmacy, Shandong Provincial Hospital Affiliated to Shandong University, Jinan, China, ${ }^{2}$ Department of Pharmacy, Nanjing Drum Tower Hospital, The Affiliated Hospital of Nanjing University Medical School, Nanjing, China,

${ }^{3}$ Minimally Invasive Urology Center, Shandong Provincial Hospital Affiliated to Shandong University, Jinan, China,

${ }^{4}$ Department of Cardiology, Shandong Provincial Hospital Affiliated to Shandong University, Jinan, China
}

Increasing evidence shows that inflammation plays a vital role in the occurrence and development of ischemia/reperfusion (I/R). Suppression of excessive inflammation can ameliorate impaired cardiac function, which shows therapeutic potential for clinical treatment of myocardial ischemia/reperfusion ( $\mathrm{Ml} / \mathrm{R})$ diseases. In this study, we investigated whether Ginkgolide C (GC), a potent anti-inflammatory flavone, extenuated $\mathrm{MI} / \mathrm{R}$ injury through inhibition of inflammation. In vivo, rats with the occlusion of the left anterior descending (LAD) coronary artery were applied to mimic MI/R injury. In vitro, primary cultured neonatal ventricular myocytes exposed to hypoxia/reoxygenation $(H / R)$ were applied to further discuss the anti-H/R injury property of GC. The results revealed that GC significantly improved the symptoms of $M I / R$ injury, as evidenced by reducing infarct size, preventing myofibrillar degeneration and reversing the mitochondria dysfunction. Moreover, histological analysis and Myeloperoxidase (MPO) activity measurement showed that GC remarkably suppressed Polymorphonuclears (PMNs) infiltration and ameliorated the histopathological damage. Furthermore, GC pretreatment was shown to improve $\mathrm{H}$ /R-induced ventricular myocytes viability and enhance tolerance of inflammatory insult, as evidenced by suppressing expression of

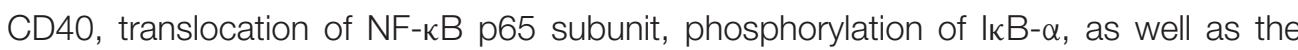
activity of IKK- $\beta$. In addition, downstream inflammatory cytokines modulated by NF$\kappa \mathrm{B}$ signaling were effectively down-regulated both in vivo and in vitro, as determined by immunohistochemistry and ELISA. In conclusion, these results indicate that GC possesses a beneficial effect against MI/R injury via inflammation inhibition that may involve suppression of CD40-NF-kB signal pathway and downstream inflammatory cytokines expression, which may offer an alternative medication for MI/R diseases.

Keywords: ginkgolide C, myocardial ischemia/reperfusion injury, inflammation, CD40, NF-kB 


\section{INTRODUCTION}

Nowadays, cardiovascular disease becomes one of the leading cause of disability and death worldwide, and one of the most popular and dangerous cardiovascular diseases is myocardial ischemia/reperfusion (MI/R) injury (Anzell et al., 2017; de Waha et al., 2017). While prompt reperfusion is favorable for myocardial salvage, ischemia/reperfusion (I/R) injury which involved a strong inflammatory response may often present itself as an adverse consequence (Liebert et al., 2017). According to the recent researches, an inflammatory response severely ignites the whole process of MI/R injury by accelerating generation of inflammatory factors and by boosting inflammatory eruption simultaneously (Montecucco et al., 2017). A disturbed inflammatory stimuli is associated with poor prognosis and increased tissue damage, may aggravate the degree of $\mathrm{I} / \mathrm{R}$ injury and it may seriously delay the recovery procedures (Pantazi et al., 2016; Yarijani et al., 2017). Therefore, restoration of cardiac dysfunction through modulation of inflammatory pathways is a favorable therapeutic strategy against MI/R diseases superimposed on other cardiovascular disease risk factors.

CD40, one member of tumor necrosis factor receptor (TNFR) family, serves as a transmembrane type I receptor (Clark, 2014). Except for immune cells, CD 40 has been similarly found on endothelial cells, myocytes, epithelial cells, fibroblasts, and monocytes (Senhaji et al., 2015). Tumor necrosis factor (TNF)- $\alpha$, Interleukin (IL)-1, Interferon (IFN)- $\gamma$, CD40 ligand (CD40L) and others inflammatory factors can regulate the expression of CD40. Then, the abundant expression of CD40 in the cytoplasmic domain will tend to TNF receptor-associated factors (TRAFs), which consequently activates different signal pathways, such as

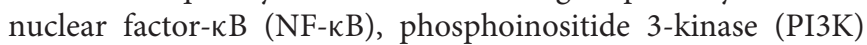
and MAPKs, in different stages of MI/R injury process (Jansen et al., 2016). Researches for near years have indicated that CD40 was closely related to the occurrence and development of many diseases, including lung ailments, inflammatory kidney disease, diabetes, coronary artery disease and arteriosclerosis disease (Aloui et al., 2014; Mansouri et al., 2016; Michel et al., 2017). However, few studies investigated the role of CD40 in the whole inflammatory process induced by MI/R injury.

Increasing evidence suggested that NF- $\kappa \mathrm{B}$ activation elevated in the MI/R-related infarct area, inflammation was suppressed when NF- $\kappa \mathrm{B}$ activation was inhibited, and cardiac preservation was provided (Zhang et al., 2017). IкB- $\alpha$, which binds to NF- $\kappa B$ p65/p50 heterodimer in the cytoplasm under the rest state, serves as an inhibitor of NF-кB. Subsequently, ІкB kinase $\beta$ (IKK- $\beta$ ) activation induces the phosphorylation and degradation of IкB- $\alpha$ resulting in the translocation of NF- $\kappa B$ from cytoplasm to nucleus, which will enhance synthesis of various inflammatory cytokines, such as TNF- $\alpha$, IL-1 $\beta$, IL-6, vascular cell adhesion molecule-1 (VCAM-1), intracellular adhesion molecule-1 (ICAM-1) and inducible nitric oxide synthase (iNOS), that act directly or indirectly to depress cardiac function (Durand and Baldwin, 2017; Nennig and Schank, 2017). However, many studies have reported that aspirin possesses the strong anti-inflammatory properties through inhibiting the transcription factor such as NF- $\mathrm{KB}$ (He et al.,
2012). Moreover, neutrophils infiltration into the post-ischemic myocardium has previously been related to the pathogenesis of I/R injury. Reperfusion accelerates the recruitment of neutrophils into the ischemic myocardium (Sivalingam et al., 2017). Thus, suppressing neutrophils infiltration and NF- $\mathrm{kB}$ pathway activation can diminish MI/R damage and possibly improve myocardial function. From this evidence, it was reasonable to speculate that NF- $\mathrm{BB}$ signal pathway played a key role throughout the whole course of MI/R injury. Therefore, potent new agents for treatment of MI/R injury via anti-inflammatory effect become an imperative requirement.

Ginkgolide C (GC, Figure 1), isolated from Ginkgo biloba leaves, is a flavone with multiple biological functions and has a long application history in clinical therapy in Asia (Huang et al., 2014). It was reported that GC exerted protection of ischemic diseases, inhibition of platelet aggregation, prevention of thrombus, anti-inflammation, and anti-allergy (Lau et al., 2013; Liou et al., 2015). However, up to now, there is little research on the relations between GC and MI/R injury, and the exact mechanism of its anti-inflammatory protective effects is still obscure to us.

Thus, taking into account activation of inflammatory response during MI/R injury, we have not only investigated the protective effect of GC both in vivo and in vitro, but also made great efforts to clarify the potential mechanism. Considering the supposed signal pathway of GC, aspirin which represents the prototype of anti-inflammatory and MI/R protective effects was selected as the positive control in this study. Left anterior descending (LAD)-occlusion-induced myocardial infarction rat model was established to imitate $\mathrm{MI} / \mathrm{R}$ in vivo. Moreover, ventricular myocytes treatment with hypoxia/reoxygenation $(H / R)$ were used to mimic $\mathrm{I} / \mathrm{R}$ injury in vitro. To further explore the mechanism, we also explored the role of CD40-NF- $\mathrm{KB}$ signal pathway in the protection of ventricular myocytes treated with $\mathrm{H} / \mathrm{R}$.

\section{MATERIALS AND METHODS}

\section{Materials and Reagents}

GC (PubChem CID: 161120), Aspirin (PubChem CID: 2244), 2, 3, 5-Triphenyltetrazolium chloride (TTC), 3-(4, 5-dimethylthiazol-2-yl)-2, 5-diphenyltetrazolium bromide (MTT) and chloral hydrate were bought from Sigma (St. Louis, MO, United States). DMEM medium (high glucose) and newborn calf serum were products of Gibco (Grand Island,

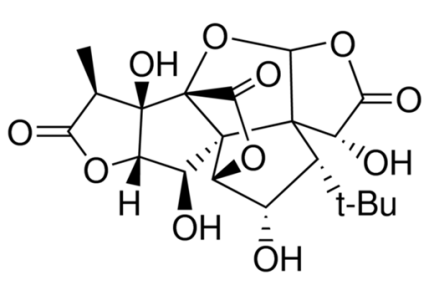

FIGURE 1 | Chemical structure of GC. 
NY, United States). Trypsin was purchased from Amresco (Solon, OH, United States). TNF- $\alpha$, IL-1 $\beta$ and IL-6 ELISA kits were bought from Sigma (St. Louis, MO, United States). Anti-ICAM-1, anti-VCAM-1, anti-iNOS, anti-CD40, anti-NF- $\kappa$ B p65, anti-p-IкB- $\alpha$, anti-IKK- $\beta$, anti- $\beta$-actin, anti-Histone, goat anti-rabbit and anti-mouse IgG antibodies were purchased from Santa Cruz (Santa Cruz, CA, United States). Nuclear-Cytosol Extraction Kit and ECL plus kit was purchased from Jiancheng (Nanjing Jiancheng Bioengineering Inc., Nanjing, China)

\section{Animals}

Adult male Sprague-Dawley rats $(250 \pm 20 \mathrm{~g})$ were provided by Experimental Animal Center of Shandong University. Rats were raised in a controlled environment (temperature $18-22^{\circ} \mathrm{C}$, humidity $40-70 \%$ ) with a $12 \mathrm{~h}$ light-dark cycle and allowed to eat and drink freely until the experiment. All the experiments were approved by the ethics committee of the Shandong University (Permission No. 2013-092).

\section{In Vivo I/R Procedure to Induce MI/R Injury in Rats}

MI/R surgery was precisely implemented according to the procedure in Figure 2A. The rats were anesthetized with $3 \%$ sodium pentobarbital (40 $\mathrm{mg} / \mathrm{kg}$, i.p.) and mechanically ventilated the lung with a rodent respirator. Holter monitoring electrocardiogram was continuously used to monitor the changes of S-T segment in order to estimate the success of surgery. After a left thoracotomy, a suture tied with a plastic tube was twined round the LAD coronary artery to form a snare for reversible $\mathrm{LAD}$ occlusion. According to procedure, transient regional myocardial ischemia for $40 \mathrm{~min}$ was realized by straining the suture, and reperfusion for $120 \mathrm{~min}$ was initiated by releasing the suture and removing the tension. The blood samples were collected before the rats were sacrificed.

Rats were randomly divided into 6 groups as follows ( $n=8$ per group): Control group, non-I/R rats received saline; $\mathrm{I} / \mathrm{R}$ group, I/R rats received saline; Aspirin group, I/R rats

A

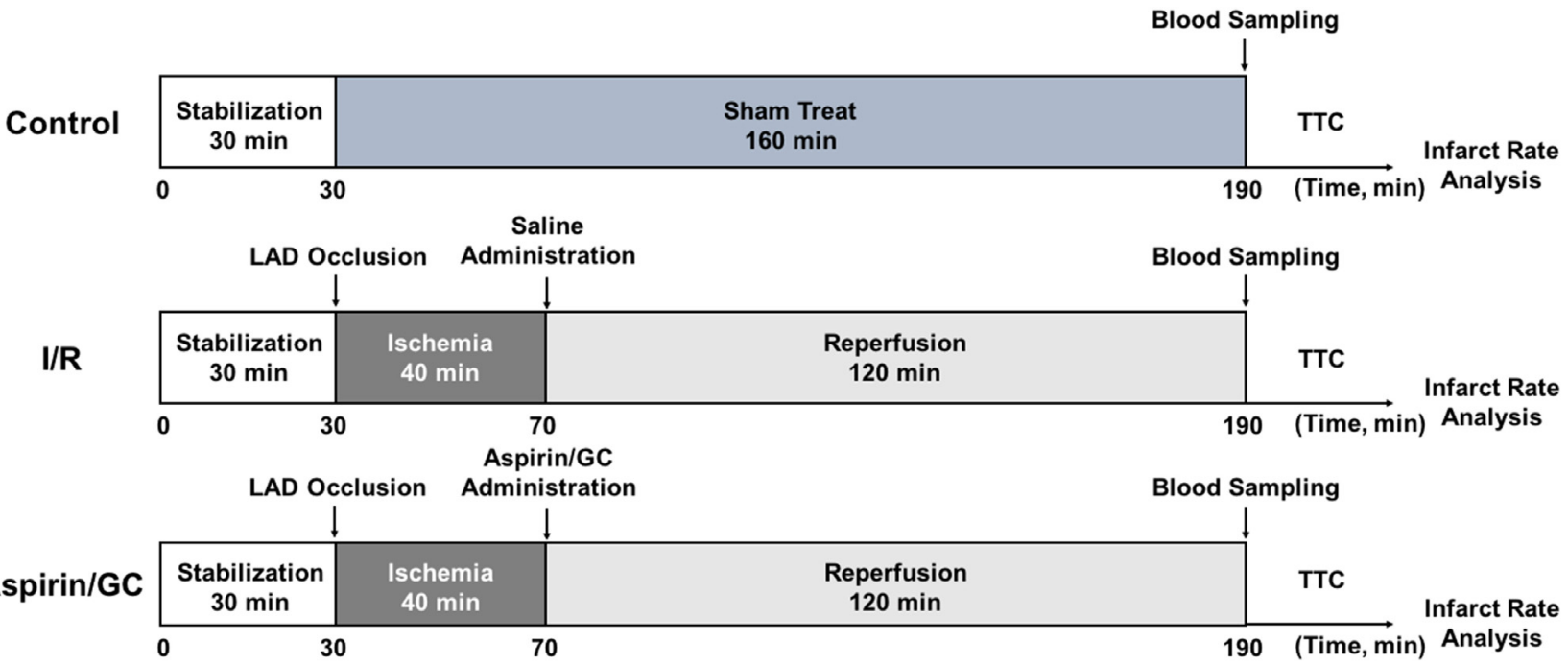

B
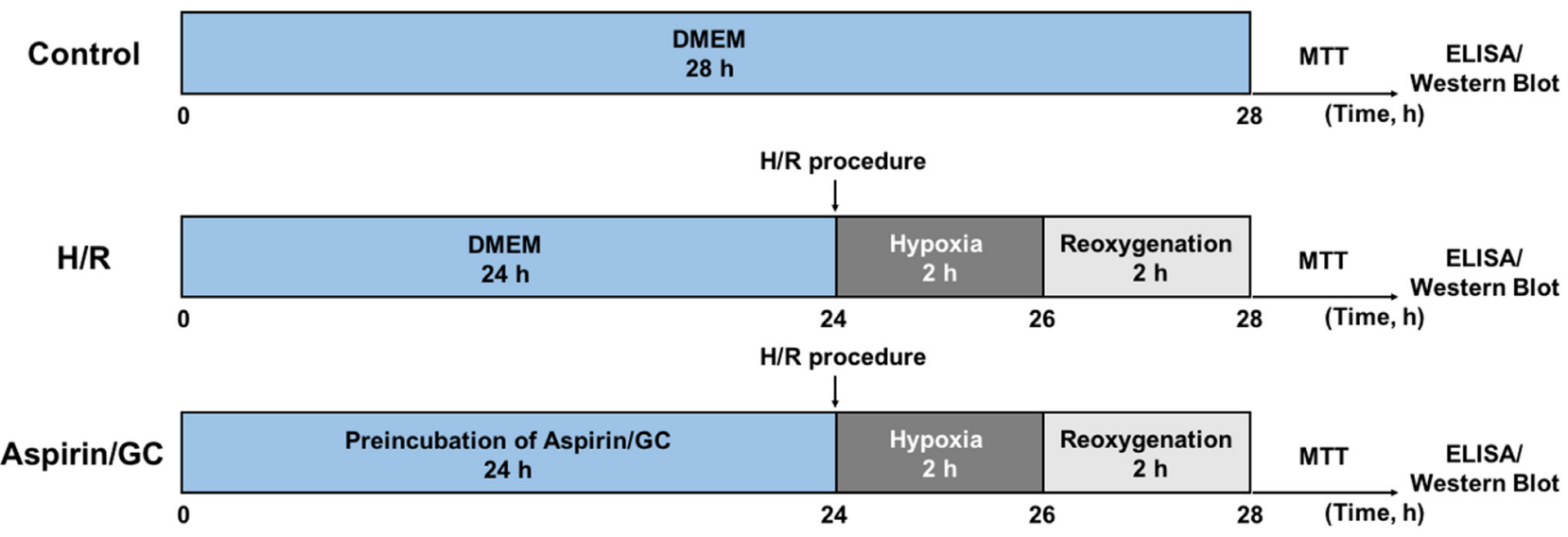

FIGURE 2 | In vivo I/R procedure (A) and in vitro H/R procedure (B). 
received $16 \mathrm{mg} / \mathrm{kg}$ of Aspirin; 8, 16, $32 \mathrm{mg} / \mathrm{kg}$ GC group, $\mathrm{I} / \mathrm{R}$ rats received $8,16,32 \mathrm{mg} / \mathrm{kg}$ of GC. Saline, Aspirin and GC were administered intraperitoneally for 7 days before I/R procedure.

\section{Measurement of Infarct Size}

Infarct size was assessed by TTC staining technique in accordance with a previous study report (Panda et al., 2017). After the completion of the experimental protocols, the hearts were removed. The left ventricle area was sectioned into five 2-3 mm-thick slices from the apex to the base. The third slice was incubated in $\mathrm{pH} 7.4,2 \%$ TTC at $37^{\circ} \mathrm{C}$ for $15 \mathrm{~min}$. Viable tissue was stained to dark red while the infarct area remained pale. Then, the area of the infarcted tissues was demarcated and measured digitally using Image-Pro Plus software (version 6.0, Media Cybernetic, United States) by computerized planimetry. Infarct size was calculated as the ratio of infarcted myocardium to the risk region $\times 100 \%$.

\section{Transmission Electron Microscope (TEM) Observation}

The third heart slice was fixed in 3.0\% glutaraldehyde buffered fixative ( $\mathrm{pH}$ 7.2) for 2-3 days. After being rinsed in PBS, the specimens were embedded in Polybed 812. Then, 90 nm-thick specimens were made and observed under electron microscope (JEM-2000EX).

\section{Histopathological Analysis and Qualitative Observation of PMNs Infiltration}

The third heart slice was stained with hematoxylin and eosin (HE) after fixation. The degree of histopathological damage was assessed with pathological scores according to the criteria reported by the previous study (Jiang et al., 2012). Scores from 0 to 5 represented no damage, mild damage, moderate damage, severe damage and highly severe damage, respectively. The total number of PMNs was also recorded simultaneously. The degree of PMNs infiltration of the area at risk was determined in three random high-power fields (HPF).

\section{Immunohistochemistry}

The expressions of ICAM-1, VCAM-1 and iNOS were examined by immunohistochemistry. In brief, the third frozen heart slice incubated with anti-ICAM-1, anti-VCAM-1, and antiiNOS antibodies overnight at $4^{\circ} \mathrm{C}$ after being blocked by $10 \%$ normal serum. Then, anti-rabbit IgG was applied to incubate the heart slices combined with primary antibody for $30 \mathrm{~min}$. Consequently, immunohisochemical staining protocol was applied to heart slice in accordance with the instructions. Images were obtained using fluorescence microscope. The optical density of positive staining area was quantified by Image-Pro Plus software (version 6.0, Media Cybernetic, United States) and the results were expressed as mean optical density mean $\pm \mathrm{SD}$.

\section{Quantitative Determination of MPO Activity}

The homogenized tissue samples were sonicated to release the MPO from tissue into the supernatant. Then, $o$-dianisidine hydrochloride and hydrogen peroxide were added, after that, MPO activity was tested at $460 \mathrm{~nm}$ according to spectrophotometer method during a period of $3 \mathrm{~min}$. The number of PMNs was counted in three random high power fields.

\section{In Vitro H/R Procedure to Induce H/R Injury in Ventricular Myocytes}

Ventricular myocytes isolated from 1 to 4-day-old SpragueDawley rats were treated with $\mathrm{H} / \mathrm{R}$ injury according to the procedure in Figure 2B. Trypsin enzymic digestion and differential attachment methods were applied to separate and purify the primary cultures of neonatal ventricular myocytes. The cells at a final density of $1 \times 10^{5} / \mathrm{mL}$ were cultured in a humidified incubator $\left(95 \%\right.$ air $/ 5 \% \mathrm{CO}_{2}$ at $\left.37^{\circ} \mathrm{C}\right)$ in DMEM supplement with $10 \%$ fetal calf serum, streptomycin $(100 \mu \mathrm{g} / \mathrm{mL})$, penicillin $(100 \mu \mathrm{g} / \mathrm{mL})$ and $0.5 \mathrm{mM}$ L-glutamine. Three days later, the cells were incubated with DMEM (low glucose) in an oxygen-free condition ( $95 \% \mathrm{~N}_{2} / 5 \% \mathrm{CO}_{2}$ ) for $2 \mathrm{~h}$ (hypoxia). And then, the cells were incubated with DMEM-F12 in a well-oxygenated condition (95\% air/5\% $\mathrm{CO}_{2}$ ) for $2 \mathrm{~h}$ (reoxygenation).

The cells were randomly divided into 6 groups as follows $(n=8$ per group): Control group, non-H/R cells cultured in DMEM medium; H/R group, cells treated with H/R procedure; Aspirin group, H/R cells preincubated with Aspirin $(10 \mu \mathrm{M})$ for $24 \mathrm{~h} ; 1$, $10,100 \mu \mathrm{M}$ GC group, H/R cells preincubated with $\mathrm{GC}(1,10$, $100 \mu \mathrm{M})$ for $24 \mathrm{~h}$.

\section{Reconstruction of CD40-Silencing Ventricular Myocytes}

When ventricular myocytes reached $80-85 \%$ confluence, the cells in control group were transfected with pGPU6/Hygro while other groups' cells were transfected with pGPU6/HygroCD40. The transfection should take $24 \mathrm{~h}$ using the GenePharma Transfection Reagent before the pretreatment of GC, Aspirin and $\mathrm{H} / \mathrm{R}$ procedure.

\section{Analysis of Cell Vitality}

Cell survival of ventricular myocytes was determined by MTT assay. After $\mathrm{H} / \mathrm{R}$ procedure, $5 \mathrm{mg} / \mathrm{mL}$ MTT was added to the medium to incubate the cells for $4 \mathrm{~h}$ at $37^{\circ} \mathrm{C}$. MTT was then removed, and cells were lysed with DMSO for $15 \mathrm{~min}$. Absorbance was tested at wavelength of $490 \mathrm{~nm}$ by a microplate reader. Results were expressed as the percent of the optical density (OD) of control cells.

\section{Detections of TNF- $\alpha$, IL-1 $\beta$, and IL- 6}

Following 120 min reperfusion, all rats were deeply anesthetized, and blood samples were obtained. Cell supernatant was collected from medium after $\mathrm{H} / \mathrm{R}$ procedure. The concentrations of TNF- $\alpha$, IL- $1 \beta$ and IL- 6 were detected by ELISA kits. 


\section{Isolation of Cytoplasmic and Nuclear Protein}

Cytoplasmic and nuclear proteins from cells were extracted by Nuclear-Cytosol Extraction Kit (Applygen Technologies Inc, Beijing, China). Cultured medium from plates was removed and astrocytes were detached with cold PBS and centrifuged at $1000 \mathrm{rpm}$ for $5 \mathrm{~min}$ at $4^{\circ} \mathrm{C}$. Pellets were resuspended in $200 \mu \mathrm{L}$ of cytosol extraction buffer $\mathrm{A}$ and incubated on ice for $10 \mathrm{~min}$. Then, $10 \mu \mathrm{L}$ cytosol extraction buffer $\mathrm{B}$ was added, incubated on ice for $1 \mathrm{~min}$ and centrifuged at $1000 \times g$ for $5 \mathrm{~min}$ at $4^{\circ} \mathrm{C}$. The pellet contains crude nuclei. The supernatant was transferred to a new tube and further centrifuged at $12,000 \times g$ for $5 \mathrm{~min}$ at $4^{\circ} \mathrm{C}$. The supernatant is cytoplasmic proteins. The crude pellet was washed once with $100 \mu \mathrm{L}$ cytosol extraction buffer $\mathrm{A}$, spined for $5 \mathrm{~min}$ at $1000 \times g$ and the supernatant was discard. Fifty microliter of cold nuclear extraction buffer was added and incubated on ice for $30 \mathrm{~min}$. Finally, samples were centrifuged at $12,000 \times g$ for $5 \mathrm{~min}$ at $4^{\circ} \mathrm{C}$ and supernatants were collected as nuclear proteins.

\section{Western Blot for ICAM-1, VCAM-1, iNOS, CD40, NF- $\kappa$ B p65, p-IкB- $\alpha$, and IKK- $\beta$ Expression in Ventricular Myocytes}

The quantity of total protein was assessed by BCA assay. Fifty microgram of protein was loaded to SDS-PAGE gel and then, transferred to a PVDF membrane. After being blocked with $5 \%$ skim milk, the membrane was incubated with primary antibody (1:800) that recognized ICAM-1, VCAM-1, iNOS, CD $40, N F-\kappa B$ p 65 , p-IкB- $\alpha$ and IKK- $\beta$ proteins. After incubation for $4 \mathrm{~h}$ at $37^{\circ} \mathrm{C}$, horseradish peroxidase-conjugated secondary antibody (1:1000) was added to the membrane and the immune complexes was determined using an ECL plus kit. Images were taken using the Gel Imaging System with Quantity One software.

\section{Statistical Analysis}

All data values were expressed as the mean \pm standard deviation (SD). Post hoc tests were used to assess the statistical significance among means. Significance of difference between groups was determined by ANOVA followed by Bonferroni correction for multiple comparisons. Results were considered to be statistically significant when $P<0.05$. All statistical figures were performed using Graph Pad Prism software (Version 5.0).

\section{RESULTS}

\section{GC Relieved the Outcomes of MI/R-Induced Inflammatory Injury GC Reduced Infarct Size in MI/R Rats}

As shown in Figure 3, infarct size as a percentage of area at risk was $44.1 \pm 5.5 \%(P<0.01$ vs. control group $)$ in the I/R group, whereas administration with $8,16,32 \mathrm{mg} / \mathrm{kg}$ GC dose-dependently decreased infarct size to $37.1 \pm 4.9 \%$

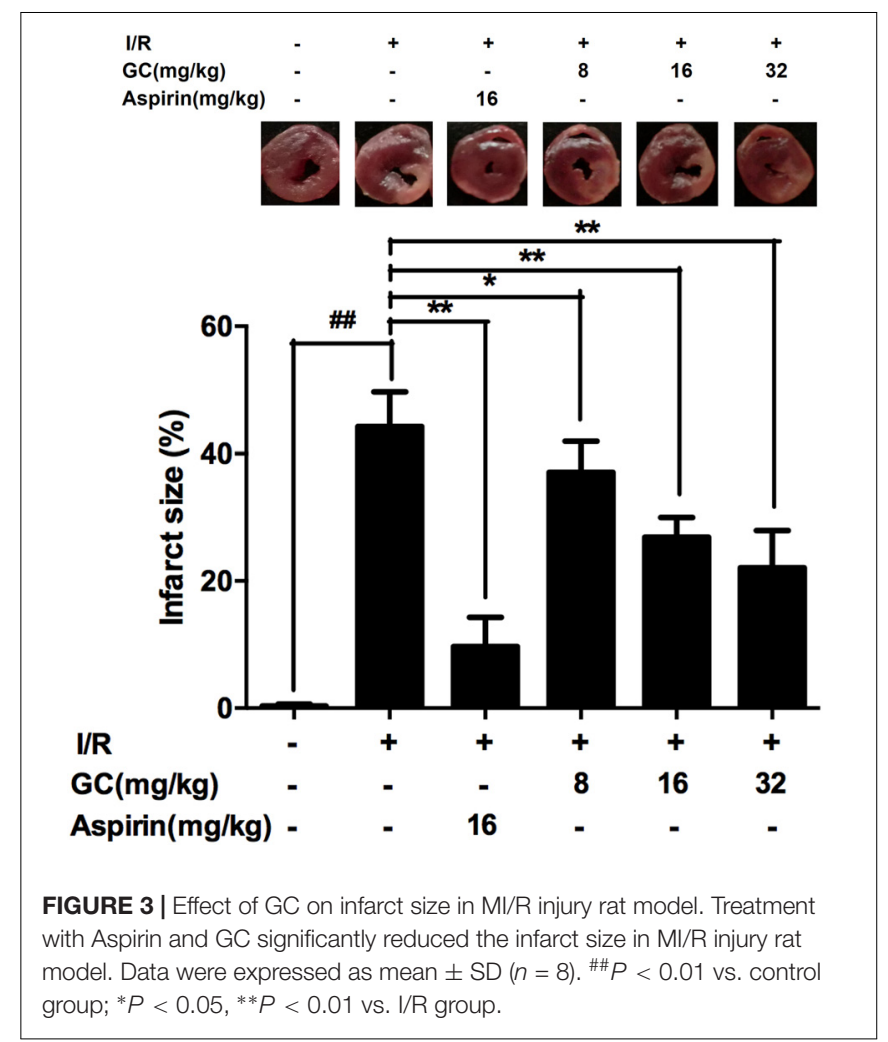

$(P<0.05), 26.9 \pm 3.1 \%(P<0.01)$, and $22.1 \pm 5.8 \%(P<0.01)$, respectively, compared with the $\mathrm{I} / \mathrm{R}$ group. The infarct size of rats after administrated with $16 \mathrm{mg} / \mathrm{kg}$ Aspirin decreased to $9.7 \pm 4.6 \%(P<0.01)$ compared with $\mathrm{I} / \mathrm{R}$ group. These findings strongly suggested that GC was effective at ameliorating MI/R injury.

\section{GC Improved Cardiac Ultrastructural Characterization in MI/R Rats}

In the present study, mitochondrial alignment and myofibrillar banding appeared normal in the control group (Figure 4A1). However, in I/R group (Figure 4A2), mitochondria became swelling and degeneration, crista fragmentation and nuclear stained deeper. Z-band disalignment and myofibrillar degeneration occurred. There was no significant change of mitochondria and myofibrillar in Aspirin group (Figure 4A3). In $8 \mathrm{mg} / \mathrm{kg}$ GC group (Figure 4A4), damages on mitochondria and formation vacuoles alleviated. In $16 \mathrm{mg} / \mathrm{kg}$ GC group (Figure 4A5), mitochondria showed mild separation of cristae without swelling and vacuolation. In $32 \mathrm{mg} / \mathrm{kg}$ GC group (Figure 4A6), only a few mitochondria showed swelling and vacuolation.

\section{GC Inhibited PMNs Infiltration in MI/R Rats}

Pathological changes were applied to further evaluate the protective effect of GC on MI/R injury. After I/R occurred, the tissue in $I / R$ injured area became necrotic, and the structure of intercalated disk and gap junction was severely impaired. However, treatment with GC and Aspirin could largely improve the histological features caused by I/R injury, characterized by 

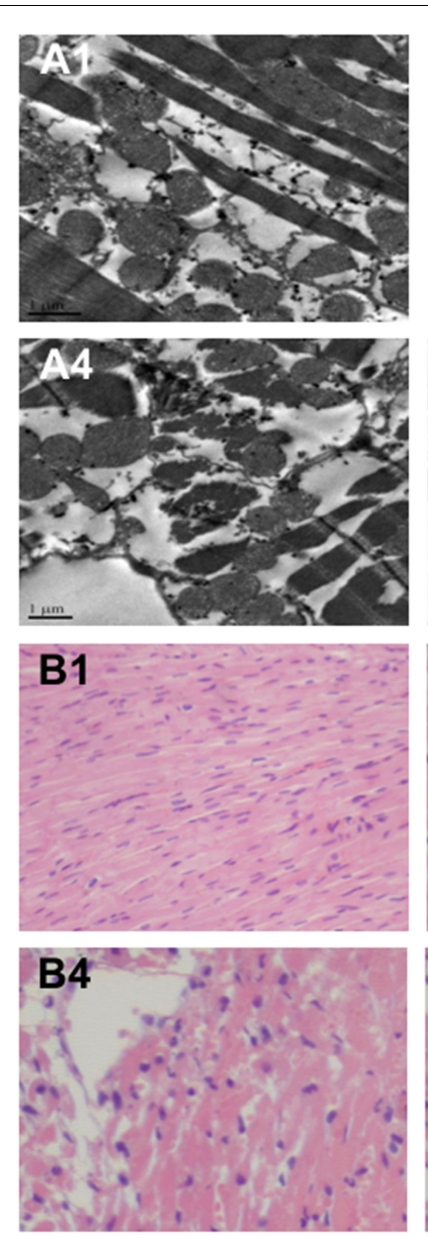
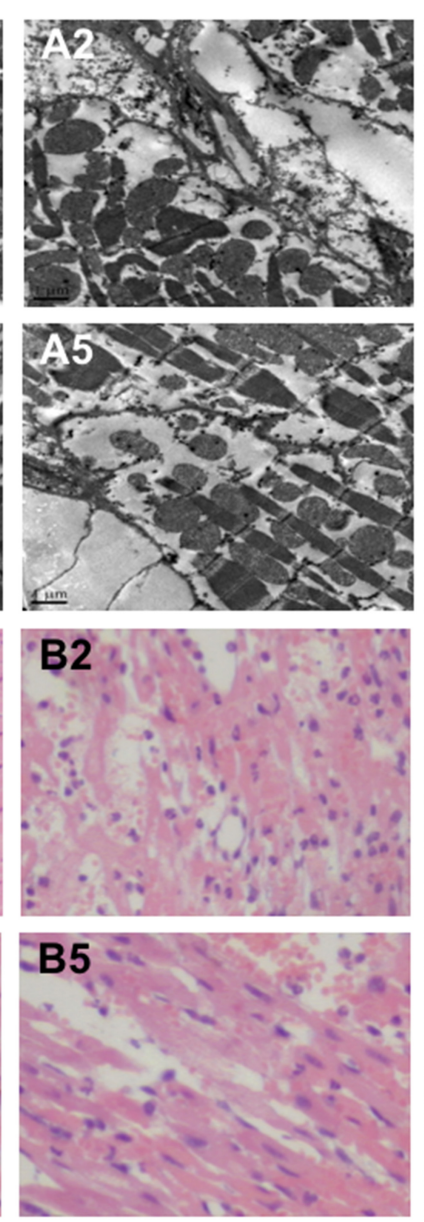
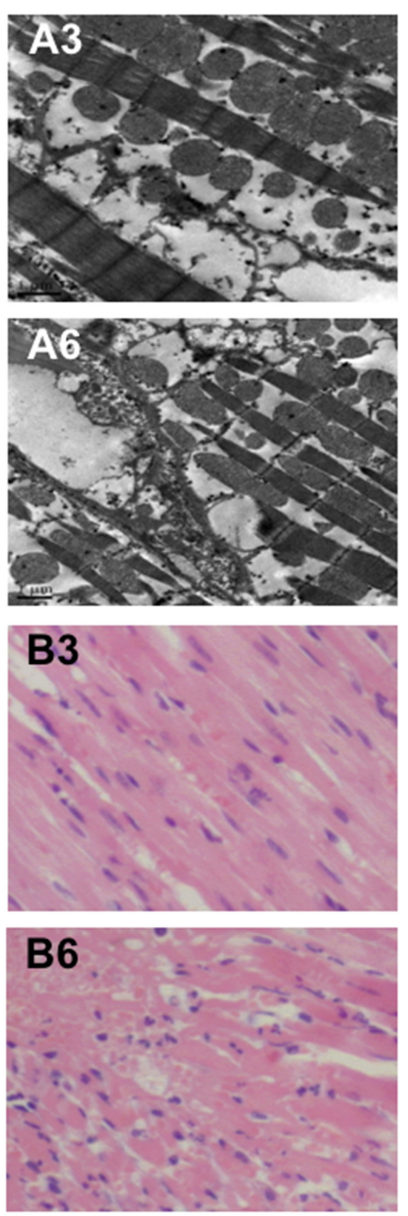

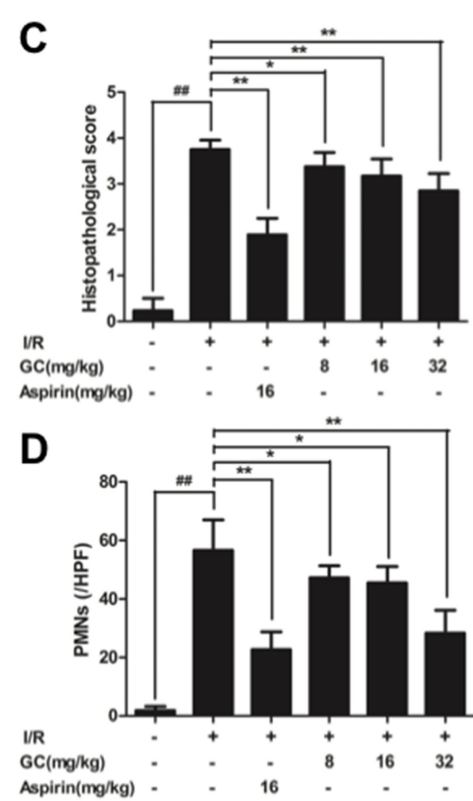

E

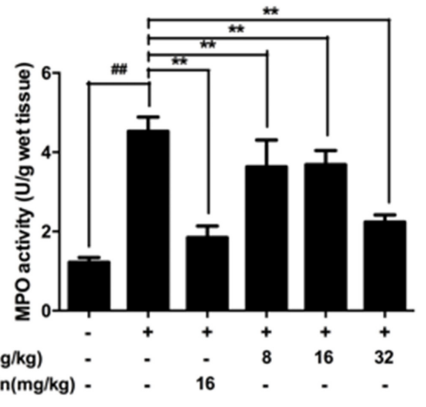

FIGURE 4 | Effects of GC on the ultrastructure of myocardial tissue, histopathological changes, myocardial PMNs counting and MPO activity.

(A1-A6) Representative transmission electron microscopy (TEM) observation of myocardial tissue injury for control group (A1), l/R group (A2), I/R + 16 mg/kg Aspirin group (A3), l/R $+8 \mathrm{mg} / \mathrm{kg}$ GC group (A4), l/R + 16 mg/kg GC group (A5), l/R + 32 mg/kg GC group (A6). (B1-B6) Representative light microscopic appearance of rat myocardial histopathological morphology (HE staining; original magnification $\times 200$ ) for control group (B1), l/R group (B2), l/R + 16 mg/kg Aspirin group (B3), l/R + 8 mg/kg GC group (B4), l/R + 16 mg/kg GC group (B5), l/R + 32 mg/kg GC group (B6). (C) Effect of GC on histopathological scores, (D) effect of GC on myocardial PMNs counting and (E) effect of GC on MPO activity. The location of the histological images was taken in the infarcted area. Data were expressed as mean $\pm \mathrm{SD}(n=8)$. ${ }^{\#} P<0.01$ vs. control group; ${ }^{*} P<0.05,{ }^{* *} P<0.01$ vs. I/R group.

TABLE 1 | Effects of GC on serum inflammatory cytokines after I/R in rats.

\begin{tabular}{|c|c|c|c|c|}
\hline Group & Dose $(\mathrm{mg} / \mathrm{kg})$ & TNF- $\alpha(p g / m L)$ & IL-1 $\beta$ (pg/mL) & IL-6 (pg/mL) \\
\hline Control & & $25.50 \pm 6.91$ & $86.63 \pm 12.52$ & $29.74 \pm 5.07$ \\
\hline I/R & & $76.10 \pm 12.91^{\# \#}$ & $257.23 \pm 18.69^{\# \#}$ & $95.87 \pm 4.80^{\# \#}$ \\
\hline I/R + Aspirin & 16 & $31.41 \pm 6.99^{* *}$ & $102.92 \pm 7.31^{* *}$ & $40.87 \pm 5.29^{* *}$ \\
\hline \multirow[t]{3}{*}{$\mathrm{I} / \mathrm{R}+\mathrm{GC}$} & 8 & $63.01 \pm 6.93^{*}$ & $200.96 \pm 8.71^{* *}$ & $87.55 \pm 4.50^{*}$ \\
\hline & 16 & $49.23 \pm 7.68^{* *}$ & $156.96 \pm 13.88^{* *}$ & $67.53 \pm 5.34^{* *}$ \\
\hline & 32 & $35.79 \pm 7.23^{* *}$ & $129.58 \pm 7.05^{* *}$ & $53.58 \pm 6.01^{* *}$ \\
\hline
\end{tabular}

Values were expressed as mean $\pm S D(n=8)$. ${ }^{\#} P<0.01$ vs. control group; $* P<0.05, * * P<0.01$ vs. I/R group.

alleviative inflammatory infiltration and recoverable structure of ischemic myocardial tissue (Figures 4B1-B6). As shown in Figure 4C, the histopathological scores were also decreased significantly by $8,16,32 \mathrm{mg} / \mathrm{kg}$ GC group compared with I/R group $(P<0.05, P<0.01, P<0.01)$. Above all, the total numbers of infiltrated and adherent PMNs in GC and Aspirin groups were remarkably less compared with that of the I/R group, as well as the histopathological score (Figures 4C,D).

To quantify neutrophilic infiltration, myocardial MPO activity was subsequently investigated. Very low MPO activity $(1.21 \pm 0.05 \mathrm{U} / g$ protein) was tested in control group (Figure $4 \mathrm{E})$. 


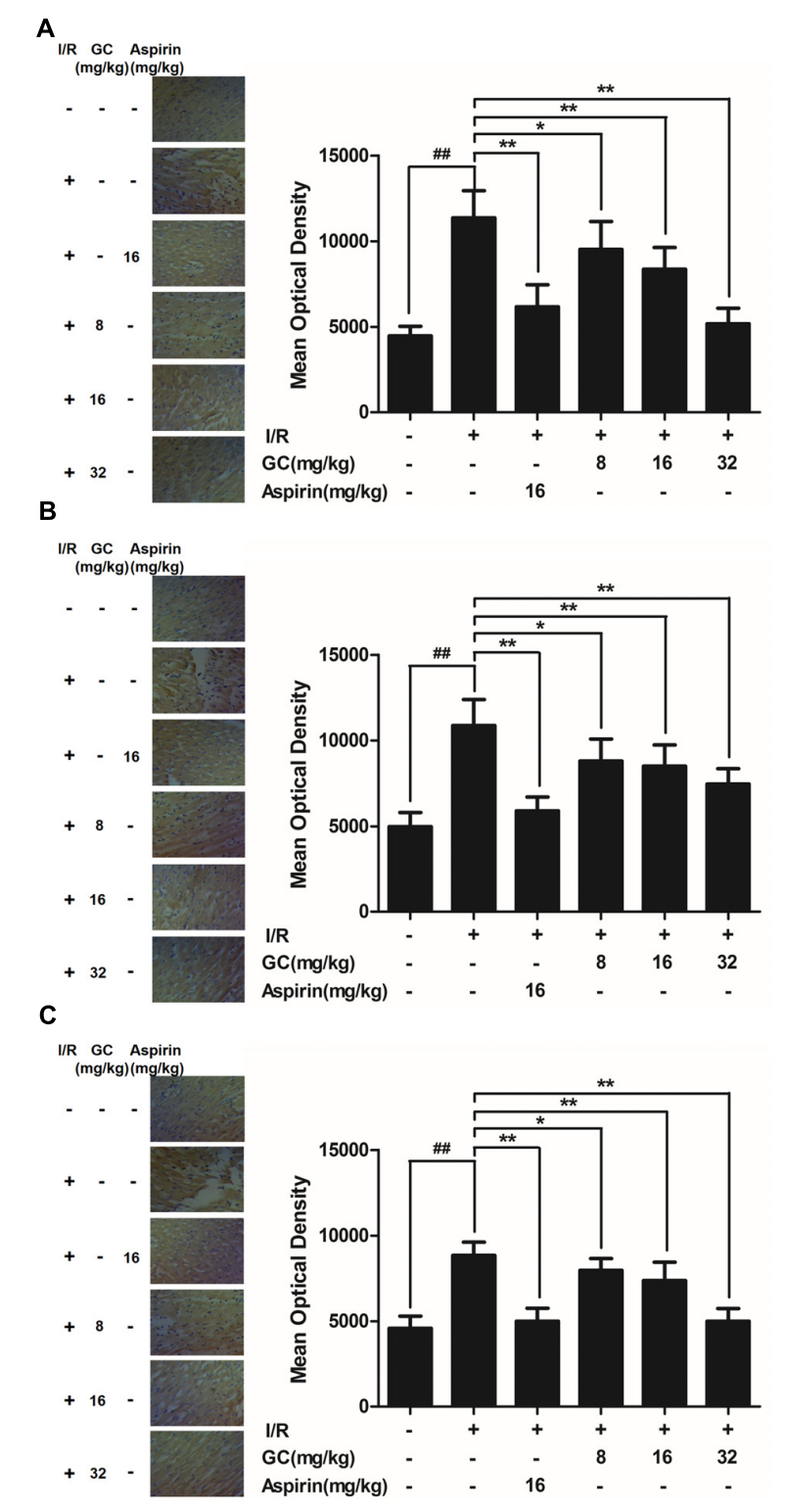

FIGURE 5 | Effect of GC on ICAM-1, VCAM-1 and iNOS expressions in myocardial tissue after I/R procedure. The tissue was observed using a microscope at a magnification $\times 400$. (A) GC decreased the expression of ICAM-1. (B) GC decreased the expression of VCAM-1. (C) GC decreased the expression of iNOS. There was a little expression of ICAM-1, VCAM-1 and iNOS in myocardial tissue of the control group. The expressions of ICAM-1, VCAM-1 and iNOS in I/R group were markedly increased. Administration of GC exhibited reduced expressions of ICAM-1, VCAM-1 and iNOS compared with the I/R group in a dose-dependent manner. Administration of Aspirin also significantly decreased the expressions of ICAM-1, VCAM-1 and iNOS compared with I/R group. The location of the histological images was taken in the infarcted area. Data were expressed as mean $\pm \mathrm{SD}(n=8)$. ${ }^{\#} P<0.01$ vs. control group; ${ }^{*} P<0.05,{ }^{*} P<0.01$ vs. I/R group.

On the contrary, elevated MPO activity was found in I/R injured group, indicating that remarkable neutrophil accumulation appeared in the I/R area of the left ventricle $(4.45 \pm 0.23 \mathrm{U} / \mathrm{g}$ protein) $(P<0.01$ vs. control group). However, compared with

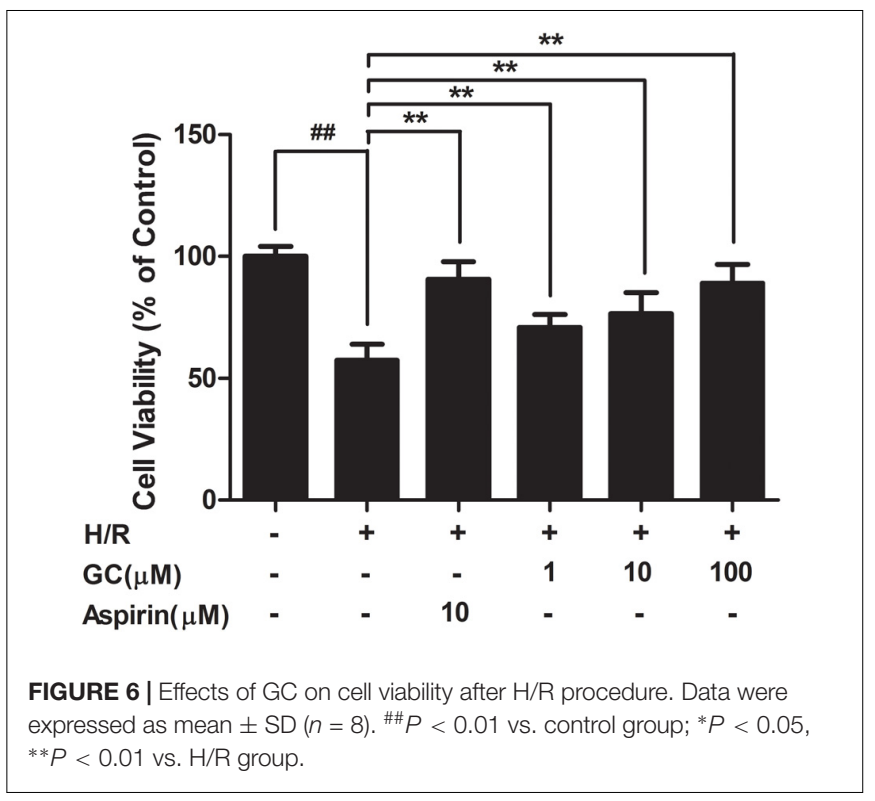

the I/R group, pretreatment with GC $8 \mathrm{mg} / \mathrm{kg}(4.20 \pm 0.22 \mathrm{U} / \mathrm{g}$ protein, $P<0.05), 16 \mathrm{mg} / \mathrm{kg}(3.75 \pm 0.30 \mathrm{U} / \mathrm{g}$ protein, $P<0.01)$, $32 \mathrm{mg} / \mathrm{kg}(2.22 \pm 0.20 \mathrm{U} / \mathrm{g}$ protein, $P<0.01)$ and Aspirin $16 \mathrm{mg} / \mathrm{kg}(1.80 \pm 0.25 \mathrm{U} / \mathrm{g}$ protein, $P<0.01)$ could prevent excessive MPO activity in myocardial tissue after I/R injury.

\section{GC Inhibited Inflammatory Cytokines Overproduction in Serum of MI/R Rats}

The concentration of inflammatory cytokines following I/R procedure in serum were detected by ELISA. Table 1 showed that I/R injury elevated the levels of TNF- $\alpha$ by 2.98 -fold $(P<0.01)$, IL-1 $\beta$ by 2.97 -fold $(P<0.01)$, and IL- 6 by $3.22-$ fold $(P<0.01)$, respectively, compared to control group. GC $(8,16,32 \mathrm{mg} / \mathrm{kg})$ dose-dependently decreased the levels of TNF- $\alpha$ by $17.2 \%(P<0.05), 35.3 \%(P<0.01)$ and $53.0 \%$ $(P<0.01)$, respectively, IL-1 $\beta$ by $21.9 \%(P<0.01), 39.0 \%$ $(P<0.01)$ and $49.6 \%(P<0.01)$, respectively, and IL-6 by $8.7 \%$ $(P<0.05), 29.6 \%(P<0.01)$ and $44.1 \%(P<0.01)$, respectively, compared with I/R group. In Aspirin group, the levels of TNF$\alpha$, IL-1 $\beta$ and IL-6 were decreased by $58.7 \%(P<0.01), 60.0 \%$ $(P<0.01)$ and $57.4 \%(P<0.01)$, respectively, compared with I/R group.

\section{GC Downregulated Overexpressions of ICAM-1,} VCAM-1, and iNOS in Myocardial Tissue of MI/R Rats Immunostaining was used to visualize the expressions of ICAM-1, VCAM-1 and iNOS in myocardial tissue. Compared with the control group, I/R procedure significantly increased the expressions of ICAM-1, VCAM-1 and iNOS (Figure 5). However, the administration of Aspirin remarkably reduced the damage to myocardium and decreased the expressions of ICAM-1, VCAM-1 and iNOS compared with I/R group. Furthermore, pretreatment with $8,16,32 \mathrm{mg} / \mathrm{kg}$ GC remarkably reduced the expressions of ICAM-1, VCAM-1 and iNOS in contrast with I/R group. These results indicated that GC could protect against I/R injury 
through inhibiting expressions of key inflammatory factors and thus inhibiting inflammation.

\section{GC Protected Ventricular Myocytes against H/R-Induced Inflammatory Injury GC Protected against Cell Insult after H/R Procedure in Ventricular Myocytes}

As shown in Figure 6, exposure of ventricular myocytes to $\mathrm{H} / \mathrm{R}$ procedure resulted in a markedly reduce in cell viability (57.4 $\pm 5.0 \%, P<0.01$ vs. control group) as assessed by MTT assay. Pretreatment with 1, 10, $100 \mu \mathrm{M}$ GC significantly increased the viability of ventricular myocytes in a concentration dependent manner $(77.3 \pm 6.9 \%, 83.5 \pm 9.6 \%, 94.2 \pm 5.0 \%, P<0.01$ vs. H/R group). Compared with $\mathrm{H} / \mathrm{R}$ group, pretreatment with Aspirin also significantly increased the cell viability $(93.3 \pm 6.7 \%$, $P<0.01)$.

\section{GC Inhibited Inflammatory Cytokines Overreleasing after H/R Procedure in Ventricular Myocytes}

As shown in Table 2, the production of TNF- $\alpha$ in H/R group were markedly increased by 8.47 -fold ( $P<0.01 \mathrm{vs.} \mathrm{control} \mathrm{group).}$ Pretreatment with $10 \mu \mathrm{M}$ Aspirin and 1, 10, $100 \mu \mathrm{M}$ GC could significantly reduce the levels of TNF- $\alpha$ by $68.3 \%(P<0.01)$, 15.1\% $(P<0.05), 22.4 \%(P<0.01)$ and $71.0 \%(P<0.01)$, respectively, compared with $\mathrm{H} / \mathrm{R}$ group. After treatment with $H / R$ procedure, the levels of IL- $1 \beta$ and IL- 6 in $H / R$ group remarkably increased by 26.69 -fold $(P<0.01)$ and 12.51 -fold $(P<0.01)$ compared with control group. Pretreatment with GC at the concentration of 1,10 , and $100 \mu \mathrm{M}$ dose-dependently decreased the levels of IL- $1 \beta$ by $41.5 \%$ ( $P<0.01), 52.9 \%$ $(P<0.01)$, and $69.6 \%(P<0.01)$, respectively, and IL- 6 by $6.1 \%$ $(P<0.01)$, 30.1\% $(P<0.01)$, and 57.0\% $(P<0.01)$, respectively, compared with $\mathrm{H} / \mathrm{R}$ group. In addition, $10 \mu \mathrm{M}$ Aspirin reduced the levels of IL-1 $\beta$ and IL- 6 by $77.2 \%(P<0.01)$ and $74.0 \%$ $(P<0.01)$ in contrast with $\mathrm{H} / \mathrm{R}$ group.

\section{GC Inhibited the Expression Level of CD40}

As shown in Figure 7A, H/R group revealed apparently higher level of CD40 compared with control group. There was no significant difference between $\mathrm{H} / \mathrm{R}$ group and Aspirin group in CD40 expression level. Nevertheless, 1, 10, $100 \mu \mathrm{M}$ GC groups significantly decreased CD40 level in response to $H / R$ injury $(P<0.05, P<0.01, P<0.01$ vs. $\mathrm{H} / \mathrm{R}$ group $)$.

\section{GC Inhibited Overexpressions of ICAM-1, VCAM-1, and iNOS after H/R Procedure in Ventricular Myocytes}

As shown in Figures 7B-D, the expressions of ICAM-1, VCAM1 , and iNOS in ventricular myocytes significantly elevated to about 8.16-fold $(P<0.01)$, 3.06-fold $(P<0.01)$, and 6.74-fold $(P<0.01)$ after $\mathrm{H} / \mathrm{R}$ procedure, compared with control group. While pretreated with GC $(1,10,100 \mu \mathrm{M})$ exhibited reduced expressions of ICAM-1 by $19.1 \%(P<0.05), 41.3 \%(P<0.01)$ and $85.5 \%(P<0.01)$, VCAM-1 by $27.2 \%(P<0.05), 46.1 \%(P<0.01)$ and $54.3 \%(P<0.01)$ and iNOS by $11.8 \%(P>0.05), 43.8 \%$ $(P<0.01)$ and $60.0 \%(P<0.01)$ compared with $\mathrm{H} / \mathrm{R}$ group in a concentration-dependent manner. In addition, $10 \mu \mathrm{M}$ Aspirin reduced the levels of ICAM-1, VCAM-1 and iNOS by $82.4 \%$ $(P<0.01), 59.3 \%(P<0.01)$ and $70.1 \%(P<0.01)$, respectively, compared with $\mathrm{H} / \mathrm{R}$ group.

\section{GC Inhibited NF-кB p65 Translocation from Cytosol to Nucleus}

Activation of NF- $\kappa \mathrm{B}$ pathway is thought to be a key signaling event involved in the pathogenesis of MI/R. To determine whether GC inhibited H/R-induced cardiac NF- $\kappa \mathrm{B}$ activation, we first examined the translocation of NF- $\kappa \mathrm{B}$ p65 from cytoplasm to the nucleus by western blot. As shown in Figures 7E,F, a relatively high level of NF- $\mathrm{B}$ p 65 appeared in the cytoplasm of cells but low levels in nucleus in control group. Translocation of p65 from the cytosol into nucleus was evident in H/R group, whereas $10 \mu \mathrm{M}$ Aspirin blocked this effect. As expected, such nuclear translocation was also concentration-dependently decreased by pretreatment of GC $(1,10,100 \mu \mathrm{M})$. These results confirmed our hypothesis that GC was able to inhibit the translocation of p65 subunit to nucleus upon an inflammatory stimulus such as $\mathrm{H} / \mathrm{R}$ injury.

\section{GC Inhibited IKB- $\alpha$ Phosphorylation and IKK- $\beta$ Activity}

$\mathrm{NF}-\kappa \mathrm{B}$ translocation is preceded by the phosphorylation and

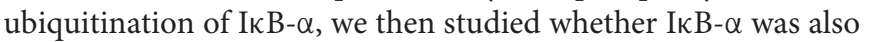
in relation to the effect of GC on NF- $\kappa B$ pathway activation. First, we checked whether the total IкB- $\alpha$ had degradated. The results showed that the total I $\mathrm{B}-\alpha$ in each group was not different (Figure 7G). However, the level of $\mathrm{p}-\mathrm{I} \kappa \mathrm{B}-\alpha$ in $\mathrm{H} / \mathrm{R}$ group remarkably increased by 4.08 -fold $(P<0.01$ vs. control group). In our study, we found a notably inhibitory effect on the $\mathrm{H} / \mathrm{R}$-induced IкB- $\alpha$ phosphorylation in the presence of Aspirin

TABLE 2 | Effects of GC on the supernatant inflammatory cytokines of ventricular myocytes.

\begin{tabular}{|c|c|c|c|c|}
\hline Group & Concentration $(\mu \mathrm{M})$ & TNF- $\alpha$ (pg/mL) & $\mathrm{IL}-1 \beta(\mathrm{pg} / \mathrm{mL})$ & IL-6 (pg/mL) \\
\hline Control & & $6.45 \pm 1.41$ & $34.02 \pm 8.85$ & $48.94 \pm 6.82$ \\
\hline $\mathrm{H} / \mathrm{R}$ & & $54.61 \pm 9.36^{\# \#}$ & $907.96 \pm 32.08^{\# \#}$ & $612.16 \pm 41.17^{\# \#}$ \\
\hline$H / R+$ Aspirin & 10 & $17.33 \pm 3.92^{* *}$ & $207.46 \pm 81.12^{* *}$ & $159.03 \pm 9.08^{* *}$ \\
\hline \multirow[t]{3}{*}{$\mathrm{H} / \mathrm{R}+\mathrm{GC}$} & 1 & $46.38 \pm 4.08^{*}$ & $531.29 \pm 54.70^{* *}$ & $574.98 \pm 48.95^{* *}$ \\
\hline & 10 & $42.35 \pm 4.47^{* *}$ & $427.73 \pm 43.31^{* *}$ & $427.75 \pm 42.88^{* *}$ \\
\hline & 100 & $15.86 \pm 2.91^{* *}$ & $275.89 \pm 78.23^{* *}$ & $263.16 \pm 15.52^{* *}$ \\
\hline
\end{tabular}

Values were expressed as mean $\pm S D(n=8) .{ }^{\#} P<0.01$ vs. control group; ${ }^{*} P<0.05,{ }^{* *} P<0.01$ vs. H/R group. 
A
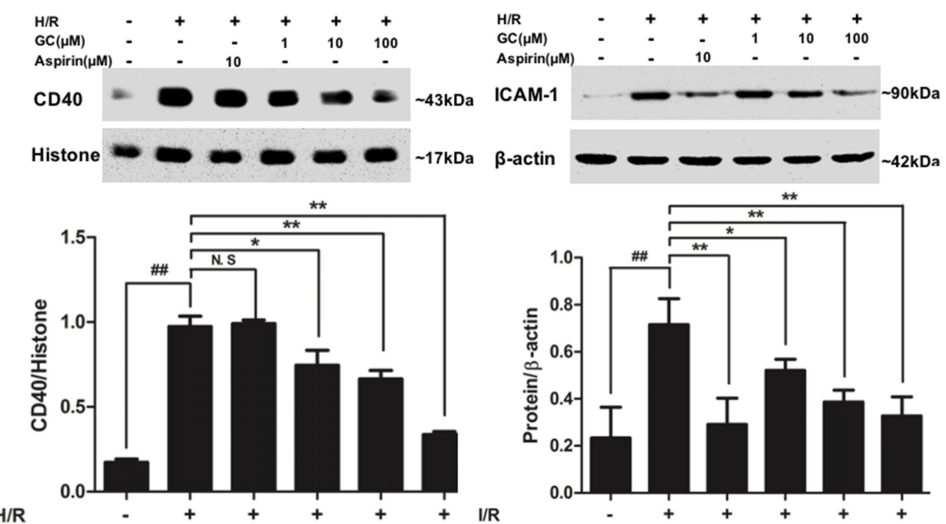

$H / R$
B

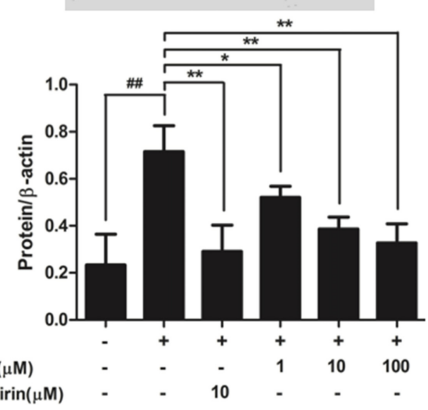

E

$H / R$
GC(MM)
Aspirim
(M)
C

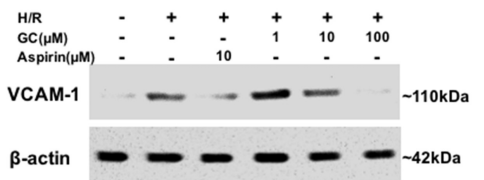

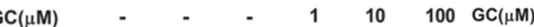

Aspirin $(\mu \mathrm{M}) \quad-\quad-10-{ }^{-}-$
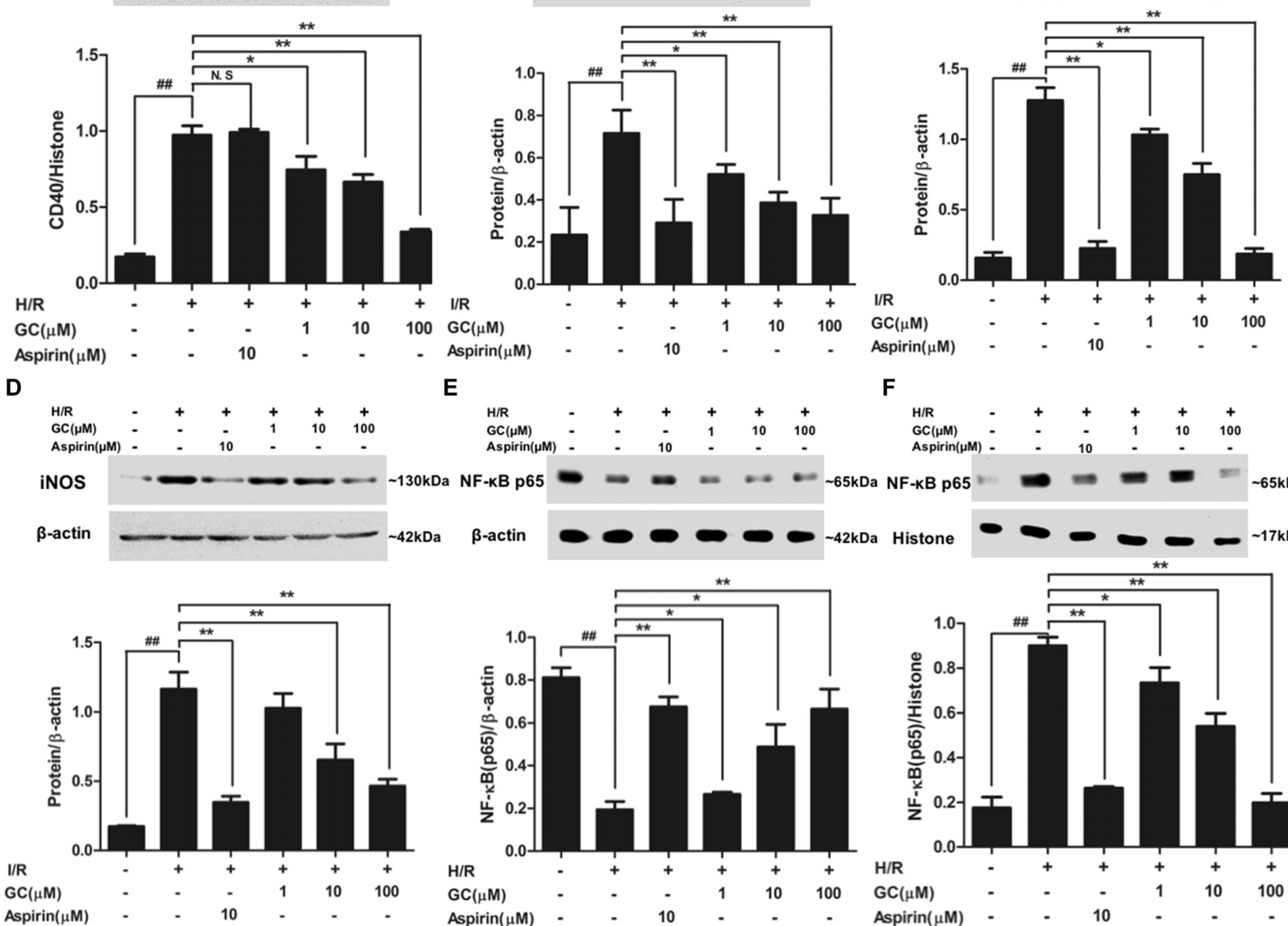

$\mathrm{GC}(\mu \mathrm{M})$

Aspirin

F

$\begin{array}{llllllc}\mathrm{H} / \mathrm{R} & - & + & + & + & + & + \\ \mathrm{GC}(\mu \mathrm{M}) & - & - & - & 1 & 10 & 100 \\ \mathrm{Aspirin}(\mu \mathrm{M}) & - & - & 10 & - & - & -\end{array}$

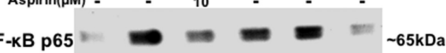

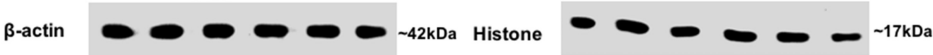
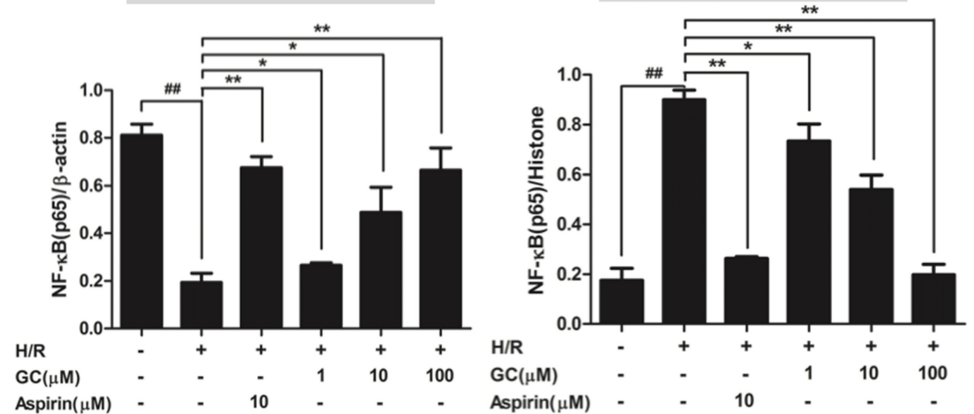

G

$\begin{array}{lcccccc}\mathrm{H} / R & - & + & + & + & + & + \\ \mathrm{GC}(\mu \mathrm{M}) & - & - & - & 1 & 10 & 100 \\ \text { Aspirin(}(\mu \mathrm{M}) & - & - & 10 & - & - & -\end{array}$

Total IкB- $\alpha=-\infty-\infty$

p-IKB-a $\cdots-$

H $\quad H$

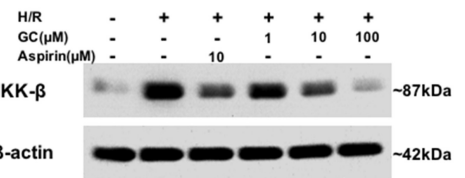

$\beta$-actin

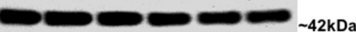

Total IkB- $\alpha$

p-IкB- $\alpha$
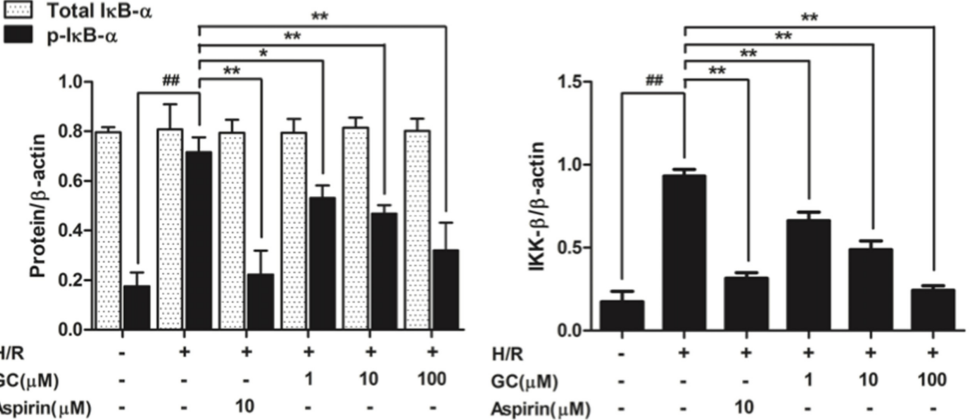

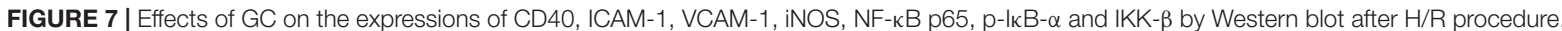

(A) GC decreased the expression of CD40. (B) GC decreased the expression of ICAM-1. (C) GC decreased the expression of VCAM-1. (D) GC decreased the expression of iNOS. GC blocked the translocation of NF-кB p65 from cytosolic (E) to nuclear (F). (G) GC down-regulated the expression of p-IкB- $\alpha$. (H) GC decreased the expression of IKK- $\beta$. CD40, ICAM-1, VCAM-1, iNOS, p-IKB- $\alpha$ and IKK- $\beta$ proteins were measured in cytosolic extract. The NF- $\mathrm{B}$ p65 protein levels were assayed separately in cytosolic and nuclear extracts. Results were expressed as Protein/reference protein ratio. Data were expressed as mean \pm SD of three independent experiments. ${ }^{\# \#} P<0.01$ vs. control group; ${ }^{*} P<0.05,{ }^{* *} P<0.01 \mathrm{vs}$. H/R group. 
and GC. The results showed that Aspirin $(10 \mu \mathrm{M})$ and GC (1, $10,100 \mu \mathrm{M})$ all reduced the expressions of $\mathrm{p}$-ІкB- $\alpha$ by $68.9 \%$ $(P<0.01), 25.8 \%(P<0.01), 34.7 \%(P<0.01)$ and $55.2 \%$ $(P<0.01)$ compared with $\mathrm{H} / \mathrm{R}$ group. These results indicated that GC could produce suppressive effect on NF- $\kappa$ B by disturbing the phosphorylation of IкB- $\alpha$.

Substantial evidence unequivocally shows that a wide variety of influential factors regulate the activity of NF- $\kappa \mathrm{B}$, especially IKK- $\beta$. Then, we investigated the effect of GC on IKK- $\beta$ activity. The results revealed a significant inhibitory effect on the H/R-induced IKK- $\beta$ activation in the presence of GC (Figure $7 \mathbf{H}$ ). The level of IKK- $\beta$ in $H / R$ group remarkably increased by 5.34-fold $(P<0.01$ vs. control group). In contrast, pretreated with Aspirin $(10 \mu \mathrm{M})$ and GC $(1,10,100 \mu \mathrm{M})$ reduced the expressions of IKK- $\beta$ (by $66.1,28.9,47.6$, and 73.8\%) $(P<0.01)$ compared with $\mathrm{H} / \mathrm{R}$ group. These findings indicate that GC could mediate IKK- $\beta$ activity.

\section{GC Failed to Alleviate H/R-Induced Ventricular Myocytes Inflammatory Injury in the Presence of CD40 Gene Silencing GC Could Not Elevate Cell Viability in the Presence of CD40 Gene Silence}

The cell viability in $\mathrm{H} / \mathrm{R}+\mathrm{CD} 40$ silencing group was significantly lower than that in control group $(P<0.01)$. After the procedure of CD40 gene silence, GC could not increase the cell viability against to H/R injury (Figure 8A).

\section{Successful and Stable CD40 Gene Silencing in Ventricular Myocytes}

The ventricular myocytes preincubated with pGPU6/Hygro expressed little CD40 after transfection in control group, and preincubation of pGPU6/Hygro-CD40 successfully and stably inhibited CD40 expression in all groups (Figure 8B).

\section{GC Could Not Inhibit Inflammatory Factors Expression in the Presence of CD40 Gene Silence}

The expressions of TNF- $\alpha$, IL- $1 \beta$, IL- 6 , ICAM-1, VCAM- 1 and iNOS in H/R + CD40 silencing group were significantly elevated compared with control group. After the procedure of CD40 gene silencing, GC could not regulate the expressions of TNF- $\alpha$, IL- $1 \beta$, IL-6, ICAM-1, VCAM-1 and iNOS (shown in Figure 8 and Table 3).

\section{GC Had No Effect on NF-кB p65 Translocation, IкB- $\alpha$ Phosphorylation and IKK- $\beta$ Activity in the Presence of CD40 Gene Silence}

$\mathrm{H} / \mathrm{R}+\mathrm{CD} 40$ silencing group procedure significantly affected the translocation level of NF- $\kappa$, activation of I $\mathrm{B}-\alpha$ phosphorylation and up-regulation of IKK- $\beta$ activity. Nonetheless, all GC groups showed no difference in NF-кB p65 translocation, I $\mathrm{B}$ - $\alpha$ phosphorylation and IKK- $\beta$ activity compared with $\mathrm{H} / \mathrm{R}+\mathrm{CD} 40$ silencing group (shown in Figure 8).

\section{DISCUSSION}

This is the first investigation studied on I/R rats subjected to GC to examine whether GC exerts significant protective effect against $\mathrm{MI} / \mathrm{R}$ injury in vivo, whether CD40 is down-regulated by GC on ventricular myocytes in response to $\mathrm{H} / \mathrm{R}$ injury in vitro, and whether NF- $\kappa$ B signal pathway plays a considerable role in the whole pathogenesis.

It has been broadly illuminated that inflammation played a vital role in the entire process of MI/R injury, and its effects refer to myocytes dysfunction, inflammatory cytokines overexpression, neutrophil accumulation and myocardium ischemia (Mohanta et al., 2014; Gragnano et al., 2017; Ma et al., 2017). Knowledge of the molecular mechanism underlying the proinflammatory processes of $\mathrm{MI} / \mathrm{R}$ injury outlined above remains incomplete. However, information does exist regarding distinct signal pathways affected, including the activation of NF- $\mathrm{B}$ signal pathway.

Ginkgo biloba is a unique tree species with no close living relatives and extracts of its leaves contain anti-inflammatory compounds including glycosides and terpenoids known as ginkgolides and bilobalides which have been reported to possess the properties of anti-cerebrovascular and anti-cardiovascular diseases (Ran et al., 2014). And, the different types of ginkgolides (A, B, C, J, and $\mathrm{M}$ ) are the main pharmaceutical effective ingredients playing major part in the established medicinal functions of G. biloba extracts (Li et al., 2017b). Among them, the pharmacologic effects of GA and GB have been studied deeply now, especially focusing on their anti-inflammatory effects applied to treatment of vascular diseases (Yao et al., 2015; Anderson and Morrow, 2017; Li et al., 2017a). Nevertheless, GC which possesses the similarity chemical structure to GA and GB has not attracted the attention of us so far. Here, GC is investigated whether it possessed a strong anti-inflammatory property, which may be one of its molecular mechanisms of how GC exerts a protective effect in the pretreatment of MI/R injury.

It is clear that lack of blood to heart results in insufficient blood and oxygen transmitted to the beat of the heart muscle, named ischemia, following by damage or dysfunction of the cardiac tissue (Hansen, 1995). In addition, in this study we investigated the effect of GC in a rat model of MI/R injury in a prophylactic approach. Here, we uncovered that pretreatment with GC at $8,16,32 \mathrm{mg} / \mathrm{kg}$ per day for 7 days protected rat from I/R insult by occlusion of LAD coronary artery, through reduction of infarct size and improvement of myocardium damage. One of the most critical and accepted indicators reflecting therapeutic effect of $\mathrm{MI} / \mathrm{R}$ injury is reduction of infarct size. In this study, our findings certified that GC was an efficient and promising target drug for prevention of $\mathrm{MI} / \mathrm{R}$ injury via significant reduction of infarct size. GC also improves the ischemic myocytes damage and inhibits reperfusion-induced energy metabolism dysfunction, at least to a certain extent via suppression of myofibrillar degeneration and restoration of myocardial ultrastructure. Moreover, our recent research clearly provides additional and strong support that G. biloba has an irreplaceable role in the therapy of diseases as a valuable herb. 

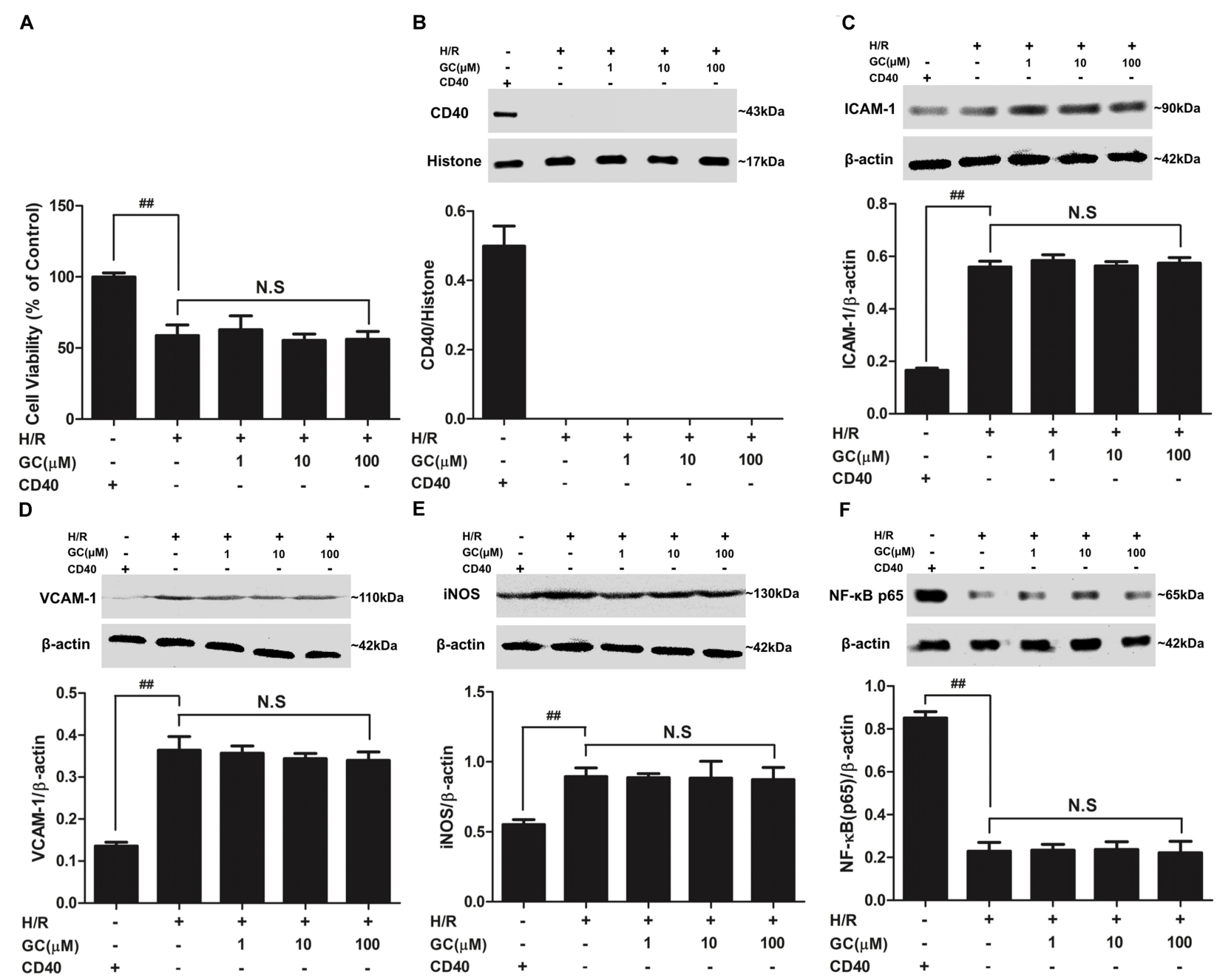

E

F
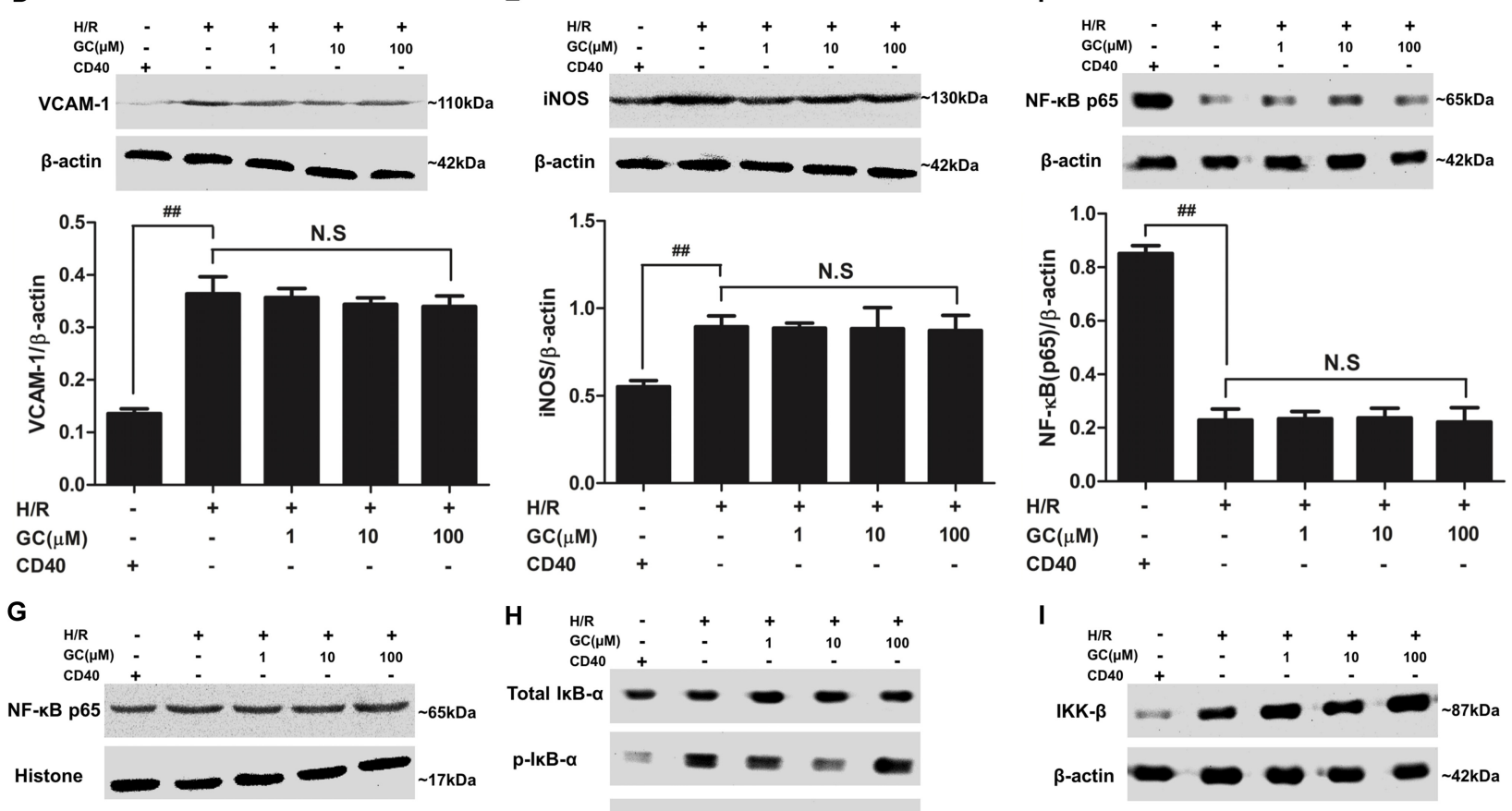

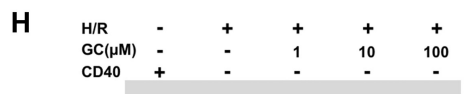
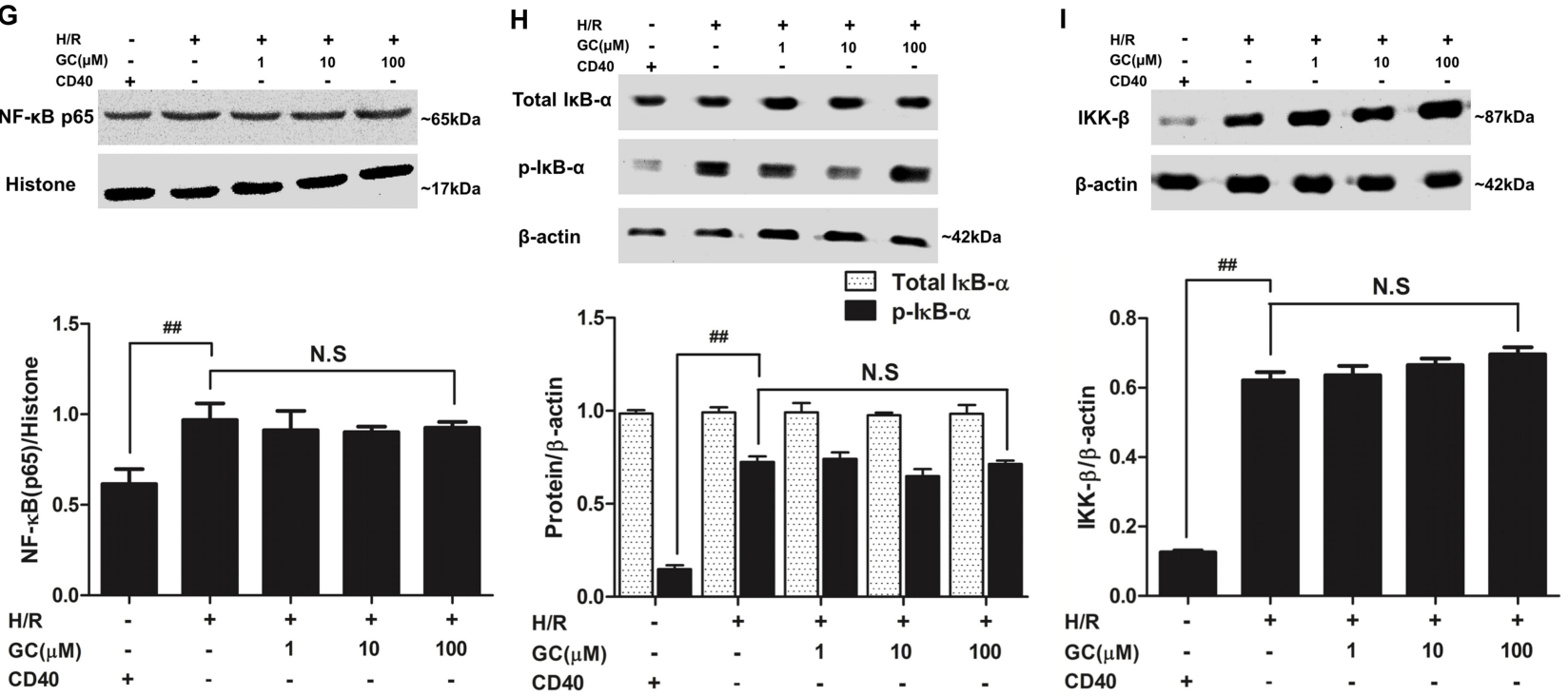

FIGURE 8 | Effects of GC on cell viability (A) and the expressions of (B) CD40, (C) ICAM-1, (D) VCAM-1, (E) iNOS, (F) cytoplasm NF-kB p65, (G) nucleus NF-kB p65, (H) p-IKB- $\alpha$ and (I) IKK- $\beta$ by Western blot after CD40 silencing procedure. Results were expressed as Protein/reference protein ratio. Data were expressed as mean $\pm \mathrm{SD}$ of three independent experiments. ${ }^{\# \#} P<0.01$ vs. control group; ${ }^{*} P<0.05,{ }^{* *} P<0.01$ vs. H/R group. 
TABLE 3 | Effects of GC on the supernatant inflammatory cytokines of ventricular myocytes under the condition of CD40 gene silencing.

\begin{tabular}{lcccc}
\hline Group & Concentration $(\boldsymbol{\mu} \mathbf{M})$ & TNF- $\boldsymbol{\alpha}(\mathbf{p g} / \mathbf{m L})$ & $\mathbf{I L - 1} \boldsymbol{\beta}(\mathbf{p g} / \mathbf{m L})$ & $\mathbf{I L - 6}(\mathbf{p g} / \mathbf{m L})$ \\
\hline Control & & $6.64 \pm 0.94$ & $35.40 \pm 7.11$ & $48.42 \pm 9.09$ \\
$\mathrm{H} / \mathrm{R}+\mathrm{CD} 40^{-}$ & & $70.08 \pm 11.39^{\# \#}$ & $1036.01 \pm 105.01^{\# \#}$ & $603.53 \pm 59.57^{\# \#}$ \\
$\mathrm{H} / \mathrm{R}+\mathrm{GC}+\mathrm{CD} 40^{-}$ & 1 & $69.27 \pm 5.88$ & $1041.32 \pm 62.15$ & $600.48 \pm 107.81$ \\
& 10 & $68.88 \pm 7.47$ & $1037.66 \pm 52.72$ & $601.44 \pm 80.31$ \\
& 100 & $68.56 \pm 4.19$ & $1023.08 \pm 72.66$ & $598.30 \pm 60.98$ \\
\hline
\end{tabular}

Values were expressed as mean $\pm S D(n=8)$. ${ }^{\#} P<0.01$ vs. control group.

Importantly, neutrophils "shoot at first sight," releasing reactive oxygen species and proteases, thereby causing extensive collateral damage to the ischemic myocardium and increasing infarct size (Stakos et al., 2015). Therefore, adherence of PMNs is identified as the beginning of a series of chain reaction after $\mathrm{I} / \mathrm{R}$ injury. What is more, a large extent by PMNs infiltrating is a main inflammation response after MI/R. Activated PMNs will lead to a mass of inflammatory mediators' release, which may directly result in the necrosis and apoptosis of myocytes (Hiroi et al., 2013; Xiao et al., 2017). Suppression of PMNs infiltration diminishes $\mathrm{MI} / \mathrm{R}$ damage and potentially offers myocardial protection. In the present study, we found a significant aggravation of histopathological damage in in vivo model, indicating that there was a definite relationship between PMNs infiltration and MI/R injury. Whereas, administration with GC to rats could remarkably alleviate this situation that leukocyte infiltration was effectively attenuated, as determined by histopathological scores and the counting of PMNs. In addition, MPO activity which correlates closely with the number of neutrophils present in the heart was also evaluated. This result corresponds with respective histopathological scores data. Our investigation may provide a new insight: the potential use of GC as a cardio protective strategy to attenuate PMNs infiltration is clinically feasible for the prevention of MI/R injury.

Moreover, in vitro, we further applied H/R-treated ventricular myocytes to stimulate $\mathrm{I} / \mathrm{R}$ injury in vivo thereby confirming the cardioprotective property of GC. Primary cultured neonatal ventricular myocytes stimulated by $\mathrm{H} / \mathrm{R}$ were further applied to explore the anti-I/R injury property of GC in vitro. Increasing evidence suggests that inflammatory and immune responses have a profound impact on the damage process of myocytes directly exposed to $H / R$ which had a strong influence on $M I / R$ injury (Jin et al., 2017). Thus, based on the above reasons, H/R-induced ventricular myocytes in vitro model was applied to explore whether GC had protected cells from inflammatory damage caused by MI/R injury. In this study, we stated for the first time that GC increased cell viability after I/R-like insult, suggesting GC can elicit anti-MI/R injury effects by promoting tolerance and viability of cells injured by $\mathrm{H} / \mathrm{R}$-induced inflammation.

NF- $\mathrm{KB}$ could induce the transcription and expression of multiple cytokines related to immunity and inflammation and other relative gene so as to cause heart damage following pathologic process of $\mathrm{I} / \mathrm{R}$ injury (Gao et al., 2016). However, there is some evidence that inhibition of NF-кB improves left ventricle $(\mathrm{LV})$ remodeling and contributes to a decrease in cardiac dysfunction after MI/R injury (Lu et al., 2016). Moreover, an extensive body of research has demonstrated that inhibition of the indirect signal pathways mediated by NF- $\kappa B$ signaling can sharply suppress the inflammation after MI/R injury (Hayden and Ghosh, 2014; Xie et al., 2015). Furthermore, there is the certainty that phosphorylation of IкB- $\alpha$ acts as the trigger of NF-кB p50/p65 heterodimers' translocation from cytoplasm to the nucleus (Mitchell and Vargas, 2016). Many studies have shown that aspirin treatment caused a strong decrease in NF$\kappa B$ activation, inhibitor of I $\mathrm{K} B-\alpha$ phosphorylation together with translocation of NF- $\kappa B$ p 65 to nucleus and IKK- $\beta$ activation (Shi et al., 2017). And many studies have shown that aspirin could improve I/R injury indexes as a positive and meaningful drug in the treatment of cardiovascular and cerebrovascular diseases (Basili et al., 2014). So we chose aspirin as the positive control to compare with the effect of GC in order to elucidate the exact mechanism. These conclusions are supported by our studies, where $\mathrm{H} / \mathrm{R}$ group obviously elevated translocation of NF- $\kappa \mathrm{B}$ p65, indicating an increased transcriptional activity of $\mathrm{p} 65$, whereas GC and aspirin effectively reversed this activated effect. In addition, stimulation with $H / R$ procedure resulted in IкB- $\alpha$ phosphorylation and degradation, which was blocked by pretreatment with GC and aspirin. These results demonstrate that the molecular regulation of GC for I/R-induced inflammation involves in the inhibition of the NF- $\mathrm{kB}$ signal pathway, as shown by the reduction in phosphorylation of IкB- $\alpha$ and translocation of NF- $\mathrm{kB}$ p 65 .

It is now clear that NF- $\mathrm{KB}$ signaling is tightly regulated at the level of IкB phosphorylation. The IKK complex, composed of IKK $(\alpha, \beta$, and $\gamma)$, is activated by phosphorylation of IKK- $\alpha$ or IKK- $\beta$ on serine residues within their activation loops either by upstream kinases or through autophosphorylation (Kondylis et al., 2017). The activated complex goes on to phosphorylate IкB- $\alpha$, causing its ubiquitin-mediated degradation and release of the NF- $\kappa B$ subunits (Yang et al., 2017). And lots of evidence has shown that aspirin's inhibitory effect of NF- $\mathrm{KB}$ has a close relationship with downregulation of IKK- $\beta$ activity (Gao et al., 2014). Then, we detected whether GC has the similar impact like aspirin on IKK- $\beta$ activity. Interestingly, IKK- $\beta$ could also be inhibited by GC in H/R-induced intact ventricular myocytes. Therefore, we concluded that one of the anti-inflammatory targets of GC was IKK- $\beta / N F-\kappa B$ signaling.

Indeed, much evidence was given that activation of NF- $\mathrm{KB}$ occurred at the very early stages of $\mathrm{I} / \mathrm{R}$ process and then sequentially modulated the occurrence of downstream inflammatory factors (Hostager, 2007). Therefore, we performed immunostaining and ELISA analyses to check whether GC 
could inhibit the inflammatory chain reaction caused by NF- $\mathrm{B}$-dependent gene transcription after I/R procedure. Our findings revealed that GC exerted its cardioprotective effects by an inhibitory mechanism that acted on excretion of pro-inflammatory mediators (TNF- $\alpha$, IL- $1 \beta$ and IL-6) and expression of inflammatory proteins (ICAM-1, VCAM-1 and iNOS) through the NF- $\mathrm{B}$ dependent pathway of inflammation. This is in accord with the investigation that studies the effects of GC on H/R-induced ventricular myocytes model in vitro. It means that, GC plays a protective role in the management of

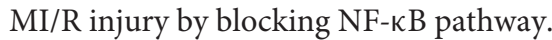

CD40 signaling is now widely accepted as a model of the non-canonical NF- $\kappa$ B pathway, which will strongly trigger its downstream signaling events (Jiang et al., 2011). More importantly, the expression of myocardial CD40 could significantly increase in the whole progression of inflammatory cardiovascular diseases (Hostager and Bishop, 2013). Therefore, CD40-mediated NF- $\mathrm{B}$ activation is thought to be responsible for the massive inflammation and tissue damage after MI/R injury, and considered to be a valuable and promising therapeutic target against $\mathrm{MI} / \mathrm{R}$ injury. Interestingly, we found that $\mathrm{GC}$ significantly down-regulated the expression of CD40 compared with $\mathrm{H} / \mathrm{R}$ group.

According to the fact, we concluded that CD40 played the vital role in regulating inflammation for ventricular myocytes in response to H/R injury. Therefore, we silence the CD40 gene in ventricular myocytes stimulated by $\mathrm{H} / \mathrm{R}$ to verify our hypothesis. As we expected, the cardioprotective effect of GC disappeared after CD40 gene was silenced. Consequently, it was evident that GC protected heart from MI/R injury through inhibiting NF- $\mathrm{B}$ pathway via CD40. It is also consistent with other investigations reporting that inhibition of $\mathrm{CD} 40$ reduces the activation of NF-кB.

Taken together, our results obtained from this work demonstrated that GC protected rat from inflammatory insult by occlusion of LAD coronary artery and inhibited H/R-induced inflammatory damage to ventricular myocytes through blocking CD40 dependent NF- $\kappa$ B signal pathway on the basis of following evidence: (1) GC improved cardiac outcomes of MI/R injury and alleviated PMNs infiltration in rats, (2) GC inhibited overproduction of inflammatory factors (TNF- $\alpha$, IL-1 $\beta$ and IL-6) and overexpression of inflammatory proteins (ICAM-1, VCAM-1

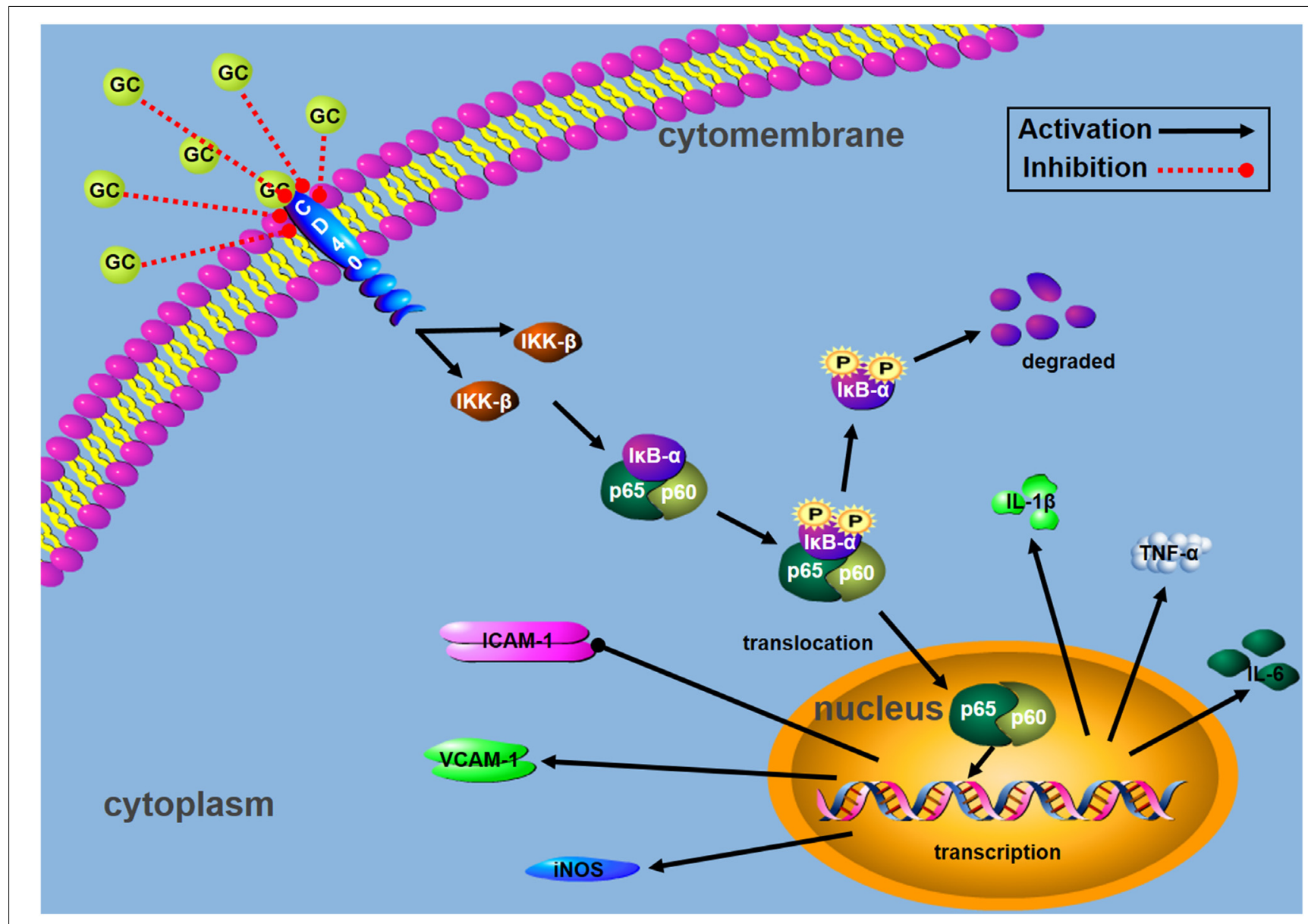

FIGURE 9 | Schematic diagram describing the mechanism in the inhibitory effect of GC on H/R-induced ventricular myocytes inflammatory injury:

$\longrightarrow$ activation; $\longrightarrow$ inhibition. 
and iNOS) both in vivo and in vitro, (3) CD40 was downregulated in ventricular myocytes in response to $H / R$ injury, and (4) such effects were dependent on regulating CD40-NF-кB pathway (Figure 9).

\section{CONCLUSION}

GC can exhibit significant cardioprotective effects through reducing infarct size, inhibiting inflammatory response, improving myocardial histological structure and alleviating PMNs infiltration during $\mathrm{I} / \mathrm{R}$ injury. Inhibition of excessive inflammation via suppressing $\mathrm{CD} 40 / \mathrm{NF}-\kappa \mathrm{B}$ signal pathway should be the key mechanism of GC in the protective of MI/R injury. Thus, GC will be a prospective and preventive agent in the management of $\mathrm{MI} / \mathrm{R}$ injury.

\section{ETHICS STATEMENT}

Animal experiments were carried out in accordance with the National Institutes of Health Guide for the Care and

\section{REFERENCES}

Aloui, C., Prigent, A., Sut, C., Tariket, S., Hamzeh-Cognasse, H., Pozzetto, B., et al. (2014). The signaling role of CD40 ligand in platelet biology and in platelet component transfusion. Int. J. Mol. Sci. 15, 22342-22364. doi: 10.3390/ ijms 151222342

Anderson, J. L., and Morrow, D. A. (2017). Acute myocardial infarction. N. Engl. J. Med. 376, 2053-2064. doi: 10.1056/NEJMra1606915

Anzell, A. R., Maizy, R., Przyklenk, K., and Sanderson, T. H. (2017). Mitochondrial quality control and disease: insights into ischemia-reperfusion injury. Mol. Neurobiol. doi: 10.1007/s12035-017-0503-9 [Epub ahead of print].

Basili, S., Tanzilli, G., Raparelli, V., Calvieri, C., Pignatelli, P., Carnevale, R., et al. (2014). Aspirin reload before elective percutaneous coronary intervention: impact on serum thromboxane b2 and myocardial reperfusion indexes. Circ. Cardiovasc. Interv. 7, 577-584. doi: 10.1161/CIRCINTERVENTIONS.113. 001197

Clark, E. A. (2014). A short history of the B-Cell-associated surface molecule CD40. Front. Immunol. 5:472. doi: 10.3389/fimmu.2014.00472

de Waha, S., Jobs, A., Eitel, I., Poss, J., Stiermaier, T., Meyer-Saraei, R., et al. (2017). Multivessel versus culprit lesion only percutaneous coronary intervention in cardiogenic shock complicating acute myocardial infarction: a systematic review and meta-analysis. Eur. Heart J. Acute Cardiovasc. Care doi: 10.1177/ 2048872617719640 [Epub ahead of print].

Durand, J. K., and Baldwin, A. S. (2017). Targeting IKK and NF-kappaB for therapy. Adv. Protein Chem. Struct. Biol. 107, 77-115. doi: 10.1016/bs.apcsb.2016.11.006

Gao, F., Si, F., Feng, S., and Yi, Q. (2016). Resistin enhances inflammatory cytokine production in coronary artery tissues by activating the NF-kappaB signaling. Biomed Res. Int. 2016:3296437. doi: 10.1155/2016/3296437

Gao, Y., Masoudi, F. A., Hu, S., Li, J., Zhang, H., Li, X., et al. (2014). Trends in early aspirin use among patients with acute myocardial infarction in China, 2001-2011: the China PEACE-Retrospective AMI study. J. Am. Heart Assoc. 3:e001250. doi: 10.1161/JAHA.114.001250

Gragnano, F., Sperlongano, S., Golia, E., and Natale, F. (2017). The role of von willebrand factor in vascular inflammation: from pathogenesis to targeted therapy. Mediators Inflamm. 2017:5620314. doi: 10.1155/2017/ 5620314

Hansen, P. R. (1995). Role of neutrophils in myocardial ischemia and reperfusion. Circulation 91, 1872-1885. doi: 10.1161/01.CIR.91.6.1872

Hayden, M. S., and Ghosh, S. (2014). Regulation of NF-kappaB by TNF family cytokines. Semin. Immunol. 26, 253-266. doi: 10.1016/j.smim.2014.05.004
Use of Laboratory Animals. All procedures involved in the use of the laboratory animals were approved by the ethics committee of Shandong University (Permission No. 2013-092).

\section{AUTHOR CONTRIBUTIONS}

RZ, DH, ZL, YZ, and PL performed the research. JL, GY, SL, BH, and JbL designed the research study. RZ and CS analyzed the data. RZ wrote and edited the paper.

\section{FUNDING}

This study was supported by grants from the National Natural Science Foundation of China (Program No. 81300964), China Postdoctoral Science Foundation (Program Nos. 2013M531611 and 2014T70648), Natural Science Foundation of Shandong Province (Program No. ZR2011HM054), and Science and Technology Development Plan Project of Shandong Province (Program No. 2014GSF118092).

He, F., Qu, F., and Song, F. (2012). Aspirin upregulates the expression of neuregulin 1 and survivin after focal cerebral ischemia/reperfusion in rats. Exp. Ther. Med. 3, 613-616. doi: 10.3892/etm.2012.450

Hiroi, T., Wajima, T., Negoro, T., Ishii, M., Nakano, Y., Kiuchi, Y., et al. (2013). Neutrophil TRPM2 channels are implicated in the exacerbation of myocardial ischaemia/reperfusion injury. Cardiovasc. Res. 97, 271-281. doi: 10.1093/cvr/ cvs332

Hostager, B. S. (2007). Roles of TRAF6 in CD40 signaling. Immunol. Res. 39, 105-114. doi: 10.1007/s12026-007-0082-3

Hostager, B. S., and Bishop, G. A. (2013). CD40-mediated activation of the NF-kappaB2 pathway. Front. Immunol. 4:376. doi: 10.3389/fimmu.2013.00376

Huang, P., Zhang, L., Chai, C., Qian, X. C., Li, W., Li, J. S., et al. (2014). Effects of food and gender on the pharmacokinetics of ginkgolides A, B, C and bilobalide in rats after oral dosing with ginkgo terpene lactones extract. J. Pharm. Biomed. Anal. 100, 138-144. doi: 10.1016/j.jpba.2014.07.030

Jansen, M. F., Hollander, M. R., Van Royen, N., Horrevoets, A. J., and Lutgens, E. (2016). CD40 in coronary artery disease: a matter of macrophages? Basic Res. Cardiol. 111:38. doi: 10.1007/s00395-016-0554-5

Jiang, W. L., Zhang, S. M., Tang, X. X., and Liu, H. Z. (2011). Protective roles of cornuside in acute myocardial ischemia and reperfusion injury in rats. Phytomedicine 18, 266-271. doi: 10.1016/j.phymed.2010.07.009

Jiang, W. L., Zhang, S. P., Zhu, H. B., and Hou, J. (2012). Cardioprotection of Asperosaponin $\mathrm{X}$ on experimental myocardial ischemia injury. Int. J. Cardiol. 155, 430-436. doi: 10.1016/j.ijcard.2011.06.010

Jin, J. L., Deng, Z. T., Lyu, R. G., Liu, X. H., and Wei, J. R. (2017). Expression changes of Notch and nuclear factor-kappaB signaling pathways in the rat heart with myocardial infarction. Zhonghua Xin Xue Guan Bing Za Zhi 45, 507-512. doi: 10.3760/cma.j.issn.0253-3758.2017.06.013

Kondylis, V., Kumari, S., Vlantis, K., and Pasparakis, M. (2017). The interplay of IKK, NF-kappaB and RIPK1 signaling in the regulation of cell death, tissue homeostasis and inflammation. Immunol. Rev. 277, 113-127. doi: 10.1111/imr. 12550

Lau, A. J., Yang, G., Rajaraman, G., Baucom, C. C., and Chang, T. K. (2013). Evaluation of Ginkgo biloba extract as an activator of human glucocorticoid receptor. J. Ethnopharmacol. 145, 670-675. doi: 10.1016/j.jep.2012.11.038

Li, Y., Wu, Y., Yao, X., Hao, F., Yu, C., Bao, Y., et al. (2017a). Ginkgolide A ameliorates LPS-induced inflammatory responses in vitro and in vivo. Int. J. Mol. Sci. 18:E794. doi: 10.3390/ijms18040794

Li, Y., Zhang, Y., Wen, M., Zhang, J., Zhao, X., Zhao, Y., et al. (2017b). Ginkgo biloba extract prevents acute myocardial infarction and suppresses 
the inflammation and apoptosis-regulating p38 mitogen-activated protein kinases, nuclear factor-kappaB and B-cell lymphoma 2 signaling pathways. Mol. Med. Rep. 16, 3657-3663. doi: 10.3892/mmr.2017. 6999

Liebert, A., Krause, A., Goonetilleke, N., Bicknell, B., and Kiat, H. (2017). A role for photobiomodulation in the prevention of myocardial ischemic reperfusion injury: a systematic review and potential molecular mechanisms. Sci. Rep. 7:42386. doi: 10.1038/srep42386

Liou, C. J., Lai, X. Y., Chen, Y. L., Wang, C. L., Wei, C. H., and Huang, W. C. (2015). Ginkgolide C suppresses adipogenesis in 3T3-L1 adipocytes via the AMPK signaling pathway. Evid. Based Complement. Alternat. Med. 2015:298635. doi: $10.1155 / 2015 / 298635$

Lu, L., Wei, P., Cao, Y., Zhang, Q., Liu, M., Liu, X. D., et al. (2016). Effect of total peony glucoside pretreatment on NF- $\mathrm{\kappa B}$ and ICAM-1 expression in myocardial tissue of rat with myocardial ischemia-reperfusion injury. Genet. Mol. Res. 15, 1-10. doi: 10.4238/gmr15048978

Ma, Y., Iyer, R. P., Jung, M., Czubryt, M. P., and Lindsey, M. L. (2017). Cardiac fibroblast activation post-myocardial infarction: current knowledge gaps. Trends Pharmacol. Sci. 38, 448-458. doi: 10.1016/j.tips.2017.03.001

Mansouri, L., Papakonstantinou, N., Ntoufa, S., Stamatopoulos, K., and Rosenquist, R. (2016). NF-kappaB activation in chronic lymphocytic leukemia: a point of convergence of external triggers and intrinsic lesions. Semin. Cancer Biol. 39, 40-48. doi: 10.1016/j.semcancer.2016.07.005

Michel, N. A., Zirlik, A., and Wolf, D. (2017). CD40L and its receptors in atherothrombosis-an update. Front. Cardiovasc. Med. 4:40. doi: 10.3389/fcrm. 2017.00040

Mitchell, S., and Vargas, J. (2016). Signaling via the NFkappaB system. Wiley Interdiscip. Rev. Syst. Biol. Med. 8, 227-241. doi: 10.1002/wsbm.1331

Mohanta, T. K., Tamboli, Y., and Zubaidha, P. K. (2014). Phytochemical and medicinal importance of Ginkgo biloba L. Nat. Prod. Res. 28, 746-752. doi: 10.1080/14786419.2013.879303

Montecucco, F., Liberale, L., Bonaventura, A., Vecchie, A., Dallegri, F., and Carbone, F. (2017). The role of inflammation in cardiovascular outcome. Curr. Atheroscler. Rep. 19:11.

Nennig, S. E., and Schank, J. R. (2017). The role of NFkB in drug addiction: beyond inflammation. Alcohol Alcohol. 52, 172-179. doi: 10.1093/alcalc/agw098

Panda, S., Kar, A., and Biswas, S. (2017). Preventive effect of agnucastoside C against isoproterenol-induced myocardial injury. Sci. Rep. 7:16146. doi: 10.1038/s41598-017-16075-0

Pantazi, E., Bejaoui, M., Folch-Puy, E., Adam, R., and Rosello-Catafau, J. (2016). Advances in treatment strategies for ischemia reperfusion injury. Expert Opin. Pharmacother. 17, 169-179. doi: 10.1517/14656566.2016.1115015

Ran, K., Yang, D. L., Chang, Y. T., Duan, K. M., Ou, Y. W., Wang, H. P., et al. (2014). Ginkgo biloba extract postconditioning reduces myocardial ischemia reperfusion injury. Genet. Mol. Res. 13, 2703-2708. doi: 10.4238/2014.April.8.14

Senhaji, N., Kojok, K., Darif, Y., Fadainia, C., and Zaid, Y. (2015). The contribution of CD40/CD40L axis in inflammatory bowel disease: an update. Front. Immunol. 6:529. doi: 10.3389/fimmu.2015.00529
Shi, C., Zhang, N., Feng, Y., Cao, J., Chen, X., and Liu, B. (2017). Aspirin inhibits IKK-beta-mediated prostate cancer cell invasion by targeting matrix metalloproteinase-9 and urokinase-type plasminogen activator. Cell Physiol. Biochem. 41, 1313-1324. doi: 10.1159/0004 64434

Sivalingam, Z., Larsen, S. B., Grove, E. L., Hvas, A. M., Kristensen, S. D., and Magnusson, N. E. (2017). Neutrophil gelatinase-associated lipocalin as a risk marker in cardiovascular disease. Clin. Chem. Lab. Med. 56, 5-18. doi: 10.1515/ cclm-2017-0120

Stakos, D. A., Kambas, K., Konstantinidis, T., Mitroulis, I., Apostolidou, E., Arelaki, S., et al. (2015). Expression of functional tissue factor by neutrophil extracellular traps in culprit artery of acute myocardial infarction. Eur. Heart J. 36, 1405-1414. doi: 10.1093/eurheartj/ehv007

Xiao, R., Xiang, A. L., Pang, H. B., and Liu, K. Q. (2017). Hyperoside protects against hypoxia/reoxygenation induced injury in cardiomyocytes by suppressing the Bnip3 expression. Gene 629, 86-91. doi: 10.1016/j.gene.2017. 07.063

Xie, Y., Xie, K., Gou, Q., and Chen, N. (2015). IkappaB kinase alpha functions as a tumor suppressor in epithelial-derived tumors through an NF-kappaBindependent pathway (Review). Oncol. Rep. 34, 2225-2232. doi: 10.3892/or. 2015.4229

Yang, C. H., Yen, T. L., Hsu, C. Y., Thomas, P. A., Sheu, J. R., and Jayakumar, T. (2017). Multi-targeting andrographolide, a novel NF-kappaB inhibitor, as a potential therapeutic agent for stroke. Int. J. Mol. Sci. 18:E1638. doi: 10.3390/ ijms18081638

Yao, X., Chen, N., Ma, C. H., Tao, J., Bao, J. A., Zong-Qi, C., et al. (2015). Ginkgo biloba extracts attenuate lipopolysaccharide-induced inflammatory responses in acute lung injury by inhibiting the COX-2 and NF-kappaB pathways. Chin. J. Nat. Med. 13, 52-58. doi: 10.1016/S1875-5364(15)60006-1

Yarijani, Z. M., Pourmotabbed, A., Pourmotabbed, T., and Najafi, H. (2017). Crocin has anti-inflammatory and protective effects in ischemia-reperfusion induced renal injuries. Iran. J. Basic Med. Sci. 20, 753-759. doi: 10.22038/IJBMS.2017. 9005

Zhang, Q., Lenardo, M. J., and Baltimore, D. (2017). 30 years of NF-kappaB: a blossoming of relevance to human pathobiology. Cell 168, 37-57. doi: 10.1016/ j.cell.2016.12.012

Conflict of Interest Statement: The authors declare that the research was conducted in the absence of any commercial or financial relationships that could be construed as a potential conflict of interest.

Copyright (c) 2018 Zhang, Han, Li, Shen, Zhang, Li, Yan, Li, Hu, Li and Liu. This is an open-access article distributed under the terms of the Creative Commons Attribution License (CC BY). The use, distribution or reproduction in other forums is permitted, provided the original author(s) and the copyright owner are credited and that the original publication in this journal is cited, in accordance with accepted academic practice. No use, distribution or reproduction is permitted which does not comply with these terms. 